\title{
A Dynamic Interval-Valued Intuitionistic Fuzzy Sets Applied to Pattern Recognition
}

\author{
Zhenhua Zhang, ${ }^{1}$ Min Wang, ${ }^{1}$ Yong Hu, ${ }^{2}$ Jingyu Yang, ${ }^{3}$ Youpei Ye, ${ }^{3}$ and Yafang $\mathrm{Li}^{4}$ \\ ${ }^{1}$ Cisco School of Informatics, Guangdong University of Foreign Studies, Guangzhou, China \\ ${ }^{2}$ School of Management, Guangdong University of Foreign Studies, Guangzhou, China \\ ${ }^{3}$ School of Computer Science and Technology, Nanjing University of Science and Technology, Nanjing, China \\ ${ }^{4}$ Laboratory of Reproductive Medicine, The First Affiliated Hospital, Southern Medical University, Guangzhou, China
}

Correspondence should be addressed to Zhenhua Zhang; zhangzhenhua@gdufs.edu.cn

Received 16 November 2012; Revised 8 April 2013; Accepted 8 April 2013

Academic Editor: Valentina E. Balas

Copyright (c) 2013 Zhenhua Zhang et al. This is an open access article distributed under the Creative Commons Attribution License, which permits unrestricted use, distribution, and reproduction in any medium, provided the original work is properly cited.

\begin{abstract}
We present dynamic interval-valued intuitionistic fuzzy sets (DIVIFS), which can improve the recognition accuracy when they are applied to pattern recognition. By analyzing the degree of hesitancy, we propose some DIVIFS models from intuitionistic fuzzy sets (IFS) and interval-valued IFS (IVIFS). And then we present a novel ranking condition on the distance of IFS and IVIFS and introduce some distance measures of DIVIFS satisfying the ranking condition. Finally, a pattern recognition example applied to medical diagnosis decision making is given to demonstrate the application of DIVIFS and its distances. The simulation results show that the DIVIFS method is more comprehensive and flexible than the IFS method and the IVIFS method.
\end{abstract}

\section{Introduction}

Professor Zadeh's paper on fuzzy sets [1] has influenced many researchers and has been applied to many application fields, such as pattern recognition [2], fuzzy reasoning [3], portfolio selection [4], and decision making [5]. In 1986, Atanassov [6] introduced membership function, nonmembership function, and hesitancy function and launched the concept of intuitionistic fuzzy sets (IFS), which generalized the FS theory. In the research field of IFS, Yager [7] discussed its cut set characteristics, some scholars (such as [8-13]) applied it to medical diagnosis, Dengfeng and Chuntian [14] and Li et al. [15] applied it to pattern recognition, and $\mathrm{Xu}[16-20]$ and Wei [21] applied it to decision making. In 1989, Atanassov and Gargov [22] proposed interval-valued intuitionistic fuzzy sets (IVIFS) based on the comparative analysis of intervalvalued fuzzy sets and IFS. Thereafter, many researchers have investigated IVIFS: some researchers discussed the cut set characteristics of IVIFS [23] based on IFS, some applied it to pattern recognition $[16,19,20,24]$, others applied it to decision making analysis [18, 20, 25-29], and Zhang et al. [29] studied interval-valued intuitionistic fuzzy reasoning. However, there are few references related to the construction of IVIFS from IFS at present. Thus, in this paper, the main objective is to study the construction and application of IVIFS from IFS.

First, we introduce a series of definitions and construction methods of DIVIFS and propose some DIVIFS models according to IFS and IVIFS. Then, on the basis of the traditional distance measures, DIVIFS is applied to pattern recognition on medical diagnosis. The simulation results show that the method introduced in this paper is more comprehensive and flexible than the IFS method and the IVIFS method. Thus, this paper can provide valuable conclusion for the application research of IFS and IVIFS. The model of DIVIFS is also useful for the generalization from fuzzy reasoning [3] and intuitionistic fuzzy reasoning to interval-valued intuitionistic fuzzy reasoning [29].

\section{Construction of DIVIFS}

Definition 1. An IFS $A$ in universe $X$ is formulated as follows $[6,22]$ :

$$
A=\left\{\left\langle x, \mu_{A}(x), v_{A}(x)\right\rangle \mid x \in X\right\}
$$


where $\mu_{A}: X \rightarrow[0,1]$ and $\nu_{A}: X \rightarrow[0,1]$ with the condition $0 \leq \mu_{A}(x)+v_{A}(x) \leq 1$ for each $x \in X . \mu_{A}(x) \in$ $[0,1]$ and $\nu_{A}(x) \in[0,1]$ denote a degree of membership and a degree of nonmembership of $x$ to $A$, respectively. For each IFS in $X$, we call $\pi_{A}(x)=1-\mu_{A}(x)-\nu_{A}(x)$ a degree of hesitancy of $x$ to $A, 0 \leq \pi_{A}(x) \leq 1$ for each $x \in X$.

Theorem 2. Let $A$ be an IFS as mentioned above; then

$$
\mu_{A}(x)-\mu_{B}(x)+\nu_{A}(x)-v_{B}(x)+\pi_{A}(x)-\pi_{B}(x)=0 .
$$

Based on Definition 1, one has Theorem 2.

Definition 3. An IVIFS $A$ in universe $X$ is stated as follows [22]:

$$
A=\left\{\left\langle x, M_{A}(x), N_{A}(x)\right\rangle \mid x \in X\right\},
$$

where $M_{A}(x)=\left[\mu_{A}^{-}(x), \mu_{A}^{+}(x)\right], N_{A}(x)=\left[\nu_{A}^{-}(x), v_{A}^{+}(x)\right]$, and $H_{A}(x)=\left[\pi_{A}^{-}(x), \pi_{A}^{+}(x)\right]$ with the conditions $M_{A}(x) \subseteq[0,1]$, $N_{A}(x) \subseteq[0,1]$, and $H_{A}(x) \subseteq[0,1]$. The interval $M_{A}(x)$, $N_{A}(x)$, and $H_{A}(x)$ denote a degree range of membership, a degree range of non-membership, and a degree range of hesitancy of $x$ to $A$, respectively. For each IVIFS in $X$, we call $\pi_{A}^{-}(x)=1-\mu_{A}^{+}(x)-\nu_{A}^{+}(x)$ lower boundary of $x$ to $A$ and $\pi_{A}^{+}(x)=1-\mu_{A}^{-}(x)-\nu_{A}^{-}(x)$ upper boundary of hesitancy of $x$ to $A$, respectively, where $0 \leq \pi_{A}^{-}(x) \leq \pi_{A}^{+}(x) \leq 1$ for each $x \in X$.

Theorem 4. Let $A$ be an IVIFS as mentioned above; then

$$
\begin{aligned}
& \mu_{A}^{+}(x)-\mu_{B}^{+}(x)+\nu_{A}^{+}(x)-\nu_{B}^{+}(x)+\pi_{A}^{-}(x)-\pi_{B}^{-}(x)=0, \\
& \mu_{A}^{-}(x)-\mu_{B}^{-}(x)+\nu_{A}^{-}(x)-\nu_{B}^{-}(x)+\pi_{A}^{+}(x)-\pi_{B}^{+}(x)=0 .
\end{aligned}
$$

\section{Based on Definition 3, one has Theorem 4.}

Definition 5. $X$ is a universe of discourse. A DIVIFS $A$ in $X$ is an object having the following form:

$$
A=\left\{\left\langle x, M_{A}(x), N_{A}(x)\right\rangle \mid x \in X\right\},
$$

where $M_{A}(x), N_{A}(x)$, and $H_{A}(x)$ are the same as Definition 3. Let $\mu_{A}^{+}(x)=\mu_{A}^{-}(x)+\alpha_{A}(x), v_{A}^{+}(x)=v_{A}^{-}(x)+\beta_{A}(x)$. Obviously, we have $\alpha_{A}(x)+\beta_{A}(x)=\pi_{A}^{+}(x)-\pi_{A}^{-}(x), \alpha_{A}(x) \geq 0, \beta_{A}(x) \geq 0$. Thus, DIVIFS is equivalent to IVIFS, and IFS is a special case of DIVIFS when $\alpha_{A}(x)=\beta_{A}(x)=0$.

From Definition 3, let all sample data be divided into three parts, $\mu_{A}^{-}(x)$ being the firm support party of event $A, v_{A}^{-}(x)$ representing the firm opposition party of event $A$, and $\pi_{A}^{+}(x)$ showing all the absent party. In the absent party, $\pi_{A}^{-}(x)$ is the firm absent party, and $\pi_{A}^{+}(x)-\pi_{A}^{-}(x)$ is the convertible absent party, in which each sample may become either the support party or the opposition party. If there is $\alpha_{A}(x)$ sample supporting event $A$ and $\beta_{A}(x)$ sample opposing event $A$, we have DIVIFS based on Definition 5. If the proportion of the absent party converted to the support party is $\lambda_{A 1}(x)$ and the proportion of the absent party converted to the opposition party is $1-\lambda_{A 1}(x)$, then the model will become a DIVIFS with single variable, where $\alpha_{A}(x)=\lambda_{A 1}(x)\left(\pi_{A}^{+}(x)-\pi_{A}^{-}(x)\right)$, $\beta_{A}(x)=\left(1-\lambda_{A 1}(x)\right)\left(\pi_{A}^{+}(x)-\pi_{A}^{-}(x)\right)$. If the firm absent party is $\pi_{A}^{-}(x)=\left(1-\lambda_{A 0}(x)\right) \pi_{A}^{+}(x)$, then the convertible absent party is $\pi_{A}^{+}(x)-\pi_{A}^{-}(x)=\lambda_{A 0}(x) \pi_{A}^{+}(x)$, and then we will get the other DIVIFS definition as follows.

Definition 6. $X$ is a universe of discourse. DIVIFS $A$ in $X$ is an object having the following form

$$
A=\left\{\left\langle x, M_{A}(x), N_{A}(x)\right\rangle \mid x \in X\right\},
$$

where $M_{A}(x), N_{A}(x)$, and $H_{A}(x)$ are the same as Definition 5 . For each $\lambda_{A 0}(x) \in[0,1]$ and for each $\lambda_{A 1}(x) \in[0,1]$, we assume that $\mu_{A}^{+}(x)=\mu_{A}^{-}(x)+\lambda_{A 0}(x) \lambda_{A 1}(x) \pi_{A}^{+}(x), \nu_{A}^{+}(x)=$ $\nu_{A}^{-}(x)+\lambda_{A 0}(x)\left(1-\lambda_{A 1}(x)\right) \pi_{A}^{+}(x)$ and that $\pi_{A}^{-}(x)=(1-$ $\left.\lambda_{A 0}(x)\right) \pi_{A}^{+}(x)$. Evidently, the DIVIFS model is also equivalent to IVIFS. If $\lambda_{A 0}(x)=0$, then DIVIFS is IFS. And if $\mu_{A}^{-}(x)=$ $\mu_{A}(x), \nu_{A}^{-}(x)=v_{A}(x), \pi_{A}^{+}(x)=\pi_{A}(x)$, then we have a special DIVIFS which is derived from IFS.

Definition 7. A DIVIFS derived from IFS is given as follows:

$$
A=\left\{\left\langle x, M_{A}(x), N_{A}(x)\right\rangle \mid x \in X\right\},
$$

where $M_{A}(x), N_{A}(x)$, and $H_{A}(x)$ are the same as Definition 5 and $\mu_{A}^{-}(x)=\mu_{A}(x), \nu_{A}^{-}(x)=\nu_{A}(x), \pi_{A}^{+}(x)=\pi_{A}(x), \mu_{A}^{+}(x)=$ $\mu_{A}(x)+\lambda_{A 0}(x) \lambda_{A 1}(x) \pi_{A}(x), v_{A}^{+}(x)=v_{A}(x)+\lambda_{A 0}(x)(1-$ $\left.\lambda_{A 1}(x)\right) \pi_{A}(x), \pi_{A}^{-}(x)=\left(1-\lambda_{A 0}(x)\right) \pi_{A}(x)$, for each $\lambda_{A 0}(x) \epsilon$ $[0,1]$ and for each $\lambda_{A 1}(x) \in[0,1]$. This DIVIFS model is also an extension of the IFS model.

\section{Conventional Distance Measures of IFS and IVIFS}

Derived from the definitions of FS, IFS, and IVIFS, some distance measures are discussed (Figure 1). Let $d=1-S$, $\Delta \mu=\mu_{A}-\mu_{B}, \Delta \nu=\nu_{A}-\nu_{B}$, and $\Delta \pi=\pi_{A}-\pi_{B}$. According to Definition 1 and Theorem $2, \Delta \mu+\Delta \nu+\Delta \pi=0$.

According to fuzzy sets [1], the distance measure of FS is composed of membership degree:

$$
d_{\mathrm{Za}}(A, B)=\left(\sum_{i=1}^{n} w_{i}|\Delta \mu|^{p}\right)^{1 / p}
$$

In [6], Atanassov introduced a distance measure (9) of IFS from membership degree and non-membership degree:

$$
d_{\mathrm{At}}(A, B)=\left(\sum_{i=1}^{n} w_{i}\left(|\Delta \mu|^{p}+|\Delta \nu|^{p}\right)\right)^{1 / p}
$$

Considering the degree range of hesitancy, Szmidt and Kacprzyk presented a novel distance measure (10) according to Definition 1 and Theorem 2 in 2000 [30]:

$$
d_{\mathrm{S}, \mathrm{K}}(A, B)=\left(\sum_{i=1}^{n} w_{i}\left(|\Delta \mu|^{p}+|\Delta \nu|^{p}+|\Delta \mu+\Delta \nu|^{p}\right)\right)^{1 / p} .
$$




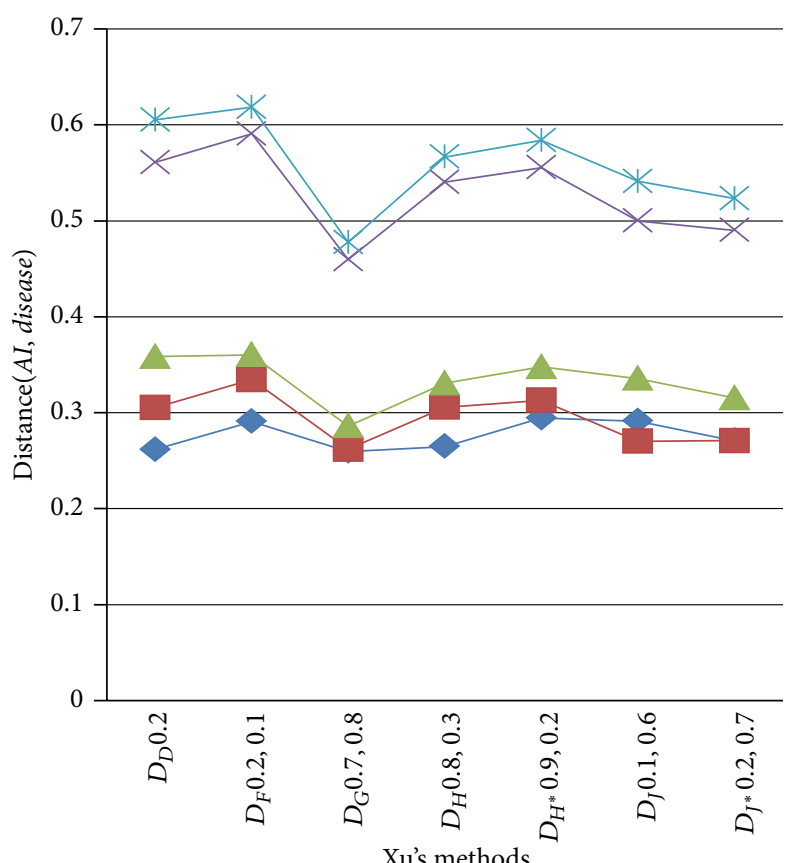

Xu's methods

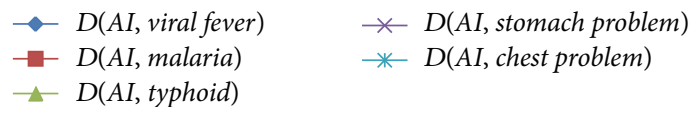

(a)

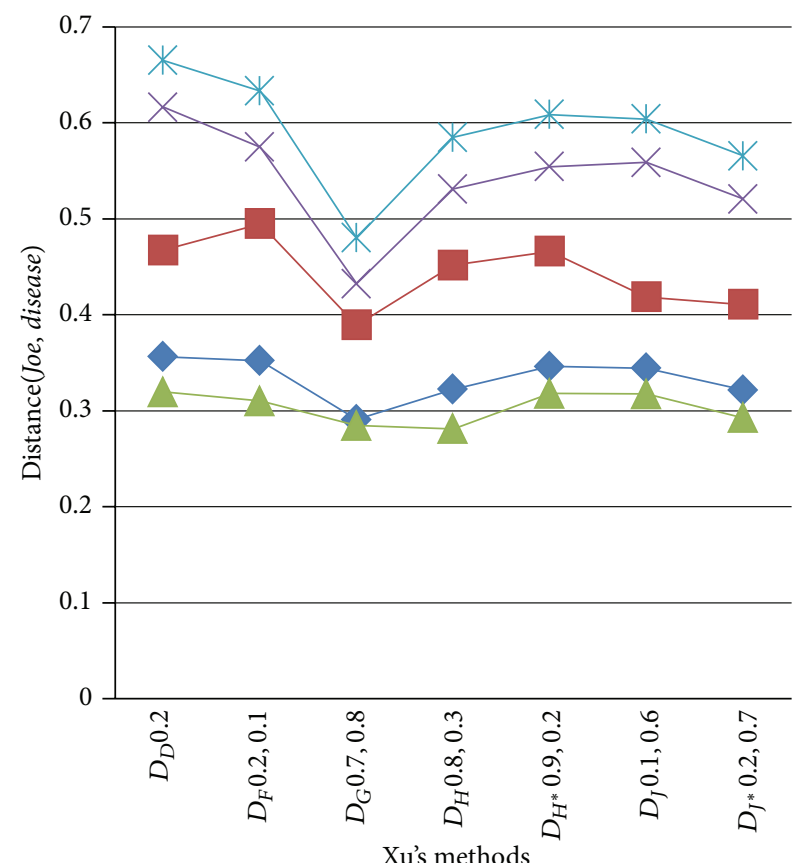

$\rightarrow D$ (Joe, viral fever $) \quad \times D$ (Joe, stomach problem $)$

- $D$ (Joe, malaria $) \quad$ * $D($ Joe, chest problem $)$

$\triangle D$ (Joe, typhoid)

(c)

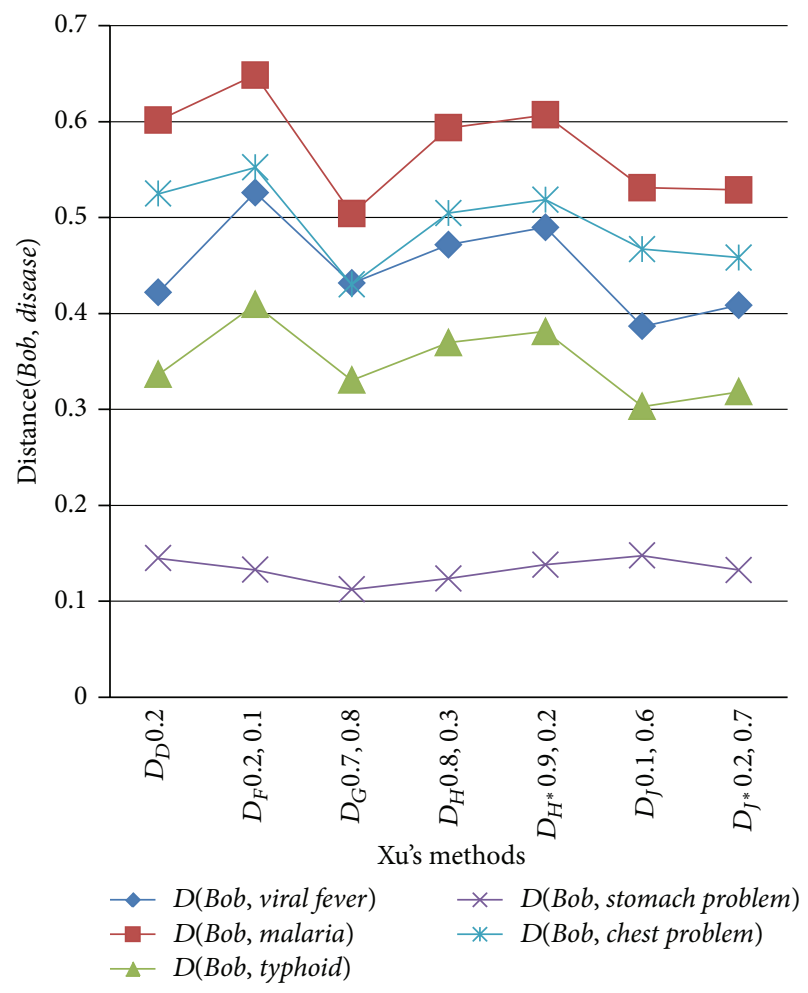

(b)

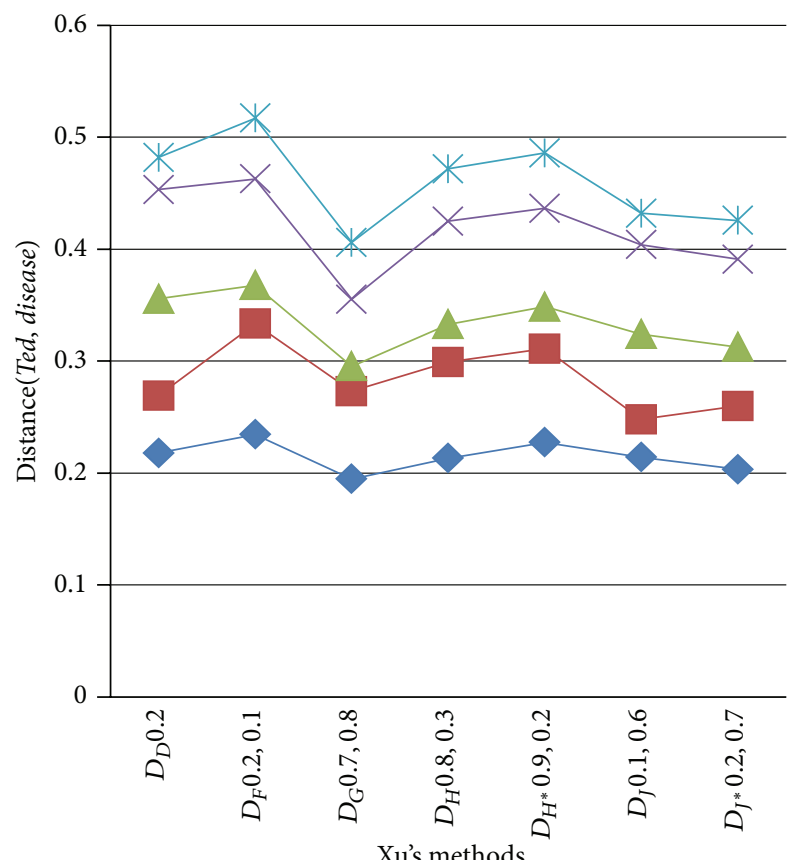

$\rightarrow D($ Ted, viral fever $) \quad \times D($ Ted, stomach problem $)$

$\rightarrow D($ Ted, malaria $) \quad$ * $D($ Ted, chest problem $)$

$\triangle D($ Ted, typhoid $)$

(d)

FIGURE 1: Distance between each patient and each disease on IFS calculated by Xu's methods. 
In [14], Dengfeng and Chuntian proposed a novel distance model as follows:

$$
d_{\mathrm{L}, \mathrm{C}}(A, B)=\left(\sum_{i=1}^{n} w_{i}|\Delta \mu-\Delta \nu|^{p}\right)^{1 / p} .
$$

At the same time, Grzegorzewski [31] and Hung and Yang [32] proposed a Hausdorff distance measure of IFS, respectively:

$$
d_{\mathrm{P}, \mathrm{H}}(A, B)=\sum_{i=1}^{n} w_{i} \max (|\Delta \mu|,|\Delta \nu|) .
$$

In 2005, Wang and Xin [33] presented a new distance measure which is made up of $d_{\mathrm{At}}$ and $d_{\mathrm{P}, \mathrm{H}}$ :

$$
d_{\mathrm{W}}(A, B)=\sum_{i=1}^{n} w_{i} \frac{|\Delta \mu|+|\Delta \nu|+2 \max (|\Delta \mu|,|\Delta \nu|)}{4} .
$$

In 2005, Liu [34] introduced some similar distance formulas as mentioned above.

In Szmidt and Kacprzyk [8-10], the ratio-based measure of similarity is defined as follows:

$$
d_{\mathrm{RB}}(A, B)=\frac{d(A, B)}{d\left(A, B^{C}\right)} .
$$

If $B$ is a set $\left(\mu_{B}(x), \nu_{B}(x), \pi_{B}(x)\right)$, Szmidt and Kacprzyk describe $B^{C}$ to be a set $\left(\nu_{B}(x), \mu_{B}(x), \pi_{B}(x)\right)$. For example, if $B$ is $\left(\mu_{B}(x)=0.7, \nu_{B}(x)=0, \pi_{B}(x)=0.3\right)$, then $B^{C}$ is $(0$, $0.7,0.3)$, and so on. And $d$ denotes a kind of distance measure about $A$ and $B$.

In 2010, Xia and Xu [20] presented some weighted distance formulas, where $k \geq 0$ and $\lambda \geq 0$ denote the weights:

$$
\begin{gathered}
d_{D_{k}}(A, B)=\left[\sum_{i=1}^{n} w_{i}\left(|(1-k) \Delta \mu-k \Delta \nu|^{p}\right)\right]^{1 / p} \\
d_{F_{k, \lambda}}(A, B) \\
=\left[0 . 5 \sum _ { i = 1 } ^ { n } w _ { i } \left(|(1-k) \Delta \mu-k \Delta \nu|^{p}+|(1-\lambda) \Delta \nu-\lambda \Delta \mu|^{p}\right.\right. \\
\left.\left.+|(1-k-\lambda)(\Delta \mu+\Delta \nu)|^{p}\right)\right]^{1 / p}, \\
k+\lambda \leq 1,
\end{gathered}
$$

$d_{G_{k, \lambda}}(A, B)$

$$
\begin{array}{r}
\left.0.5 \sum_{i=1}^{n} w_{i}\left(|k \Delta \mu|^{p}+|\lambda \Delta \nu|^{p}+|k \Delta \mu+\lambda \Delta \nu|^{p}\right)\right]^{1 / p}, \\
k+\lambda \leq 1,
\end{array}
$$

$$
\begin{array}{r}
d_{H_{k, \lambda}}(A, B) \\
=\left[0 . 5 \sum _ { i = 1 } ^ { n } w _ { i } \left(|k \Delta \mu|^{p}+|\lambda \Delta \mu-(1-\lambda) \Delta \nu|^{p}\right.\right. \\
\left.\left.+|(k-\lambda) \Delta \mu+(1-\lambda) \Delta \nu|^{p}\right)\right]^{1 / p}, \\
k+\lambda \leq 1, \\
d_{H_{k, \lambda}^{*}}(A, B) \quad\left[\begin{array}{r}
0.5 \sum_{i=1}^{n} w_{i}\left(|k \Delta \mu|^{p}+|k \lambda \Delta \mu-(1-\lambda) \Delta \nu|^{p}\right. \\
\left.\left.+|(1-\lambda)(k \Delta \mu+\Delta \nu)|^{p}\right)\right]^{1 / p}, \\
k+\lambda \leq 1,
\end{array}\right.
\end{array}
$$

$$
\begin{array}{r}
d_{J_{k, \lambda}}(A, B) \\
=\left[0 . 5 \sum _ { i = 1 } ^ { n } w _ { i } \left(|(1-k) \Delta \mu-k \Delta \nu|^{p}+|\lambda \Delta \nu|^{p}\right.\right. \\
\left.\left.+\mid(1-k) \Delta \mu+(\lambda-k) \Delta \nu)\left.\right|^{p}\right)\right]^{1 / p}, \\
k+\lambda \leq 1,
\end{array}
$$

$$
\begin{aligned}
& d_{J_{k, \lambda}^{*}}(A, B) \\
&=\left[0 . 5 \sum _ { i = 1 } ^ { n } w _ { i } \left(|(1-k) \Delta \mu-k \lambda \Delta \nu|^{p}+|\lambda \Delta \nu|^{p}\right.\right. \\
&\left.\left.+|(1-k)(\Delta \mu+\lambda \Delta \nu)|^{p}\right)\right]^{1 / p},
\end{aligned}
$$

$$
k+\lambda \leq 1 \text {. }
$$

Let $\Delta \mu^{-}=\mu_{A}^{-}-\mu_{B}^{-}, \Delta \mu^{+}=\mu_{A}^{+}-\mu_{B}^{+}, \Delta v^{-}=v_{A}^{-}-v_{B}^{-}, \Delta v^{+}=$ $v_{A}^{+}-v_{B}^{+}, \Delta \pi^{-}=\pi_{A}^{-}-\pi_{B}^{-}, \Delta \pi^{+}=\pi_{A}^{+}-\pi_{B}^{+}$. According to Definition 3 and Theorem $4, \Delta \mu^{-}+\Delta \nu^{-}+\Delta \pi^{+}=0, \Delta \mu^{+}+$ $\Delta \nu^{+}+\Delta \pi^{-}=0$. Similar to the distance measures of IFS, some scholars define some distance measures made up of $\Delta \mu^{-}, \Delta \mu^{+}$, $\Delta v^{-}, \Delta v^{+}, \Delta \pi^{-}, \Delta \pi^{+}$.

\section{Construction of Distance Measures on DIVIFS}

First, we will define a novel essential ranking property of the distance measure on IFS and IVIFS.

Definition 8. Assume that $A$ and $B$ are IFSs, and then we have the following: 
$A=B$ if and only if $\mu_{A}(x)=\mu_{B}(x), v_{A}(x)=v_{B}(x)$, $\pi_{A}(x)=\pi_{B}(x)$.

$A \subseteq B$ if and only if $\mu_{A}(x) \leq \mu_{B}(x), v_{A}(x) \geq v_{B}(x)$.

$A \subset B$ if and only if $\mu_{A}(x) \leq \mu_{B}(x)$ and $\nu_{A}(x)>v_{B}(x)$, or $\mu_{A}(x)<\mu_{B}(x)$ and $v_{A}(x) \geq v_{B}(x)$.

Definition 9. Assume that $A$ and $B$ are IVIFSs, and then we obtain the following:

$A=B$ if and only if $\mu_{A}^{-}(x)=\mu_{B}^{-}(x), \mu_{A}^{+}(x)=\mu_{B}^{+}(x)$, $v_{A}^{-}(x)=v_{B}^{-}(x), v_{A}^{+}(x)=v_{B}^{+}(x), \pi_{A}^{-}(x)=\pi_{B}^{-}(x)$, $\pi_{A}^{+}(x)=\pi_{B}^{+}(x)$.

$A \subseteq B$ if and only if $\mu_{A}^{-}(x) \leq \mu_{B}^{-}(x), \mu_{A}^{+}(x) \leq \mu_{B}^{+}(x)$, $v_{A}^{-}(x) \geq v_{B}^{-}(x), v_{A}^{+}(x) \geq v_{B}^{+}(x)$.

$A \subset B$ if and only if $A \subseteq B$ and $A \neq B$.

In general, a distance measure should conform to the following Theorem 10 according to its properties.

Theorem 10. A, B, and $C$ are IFSs; the distances among them must satisfy the following:

(P1) $d(A, B) \geq 0$ and $d(A, B)=0$ if and only if $A=B$.

(P2) $d(A, B)=d(B, A)$.

(P3) If $C \subseteq B \subseteq A$, then $d(A, C) \geq d(A, B)$ and $d(A, C \geq$ $d(B, C)$.

If $C \subset B \subset A$, then $d(A, C)>d(A, B)$ and $d(A, C)>$ $d(B, C)$.

(P4) If $D \subseteq C \subseteq B \subseteq A$, then $d(A, D) \geq d(B, C)$, and $d(A, D)=d(B, C)$ if and only if $A=B$ and $C=D$.

If $D \subset C \subset B \subset A$, then $d(A, D)>d(B, C)$.

In fact, property (P4) is derived from property (P3). According to (P3), one has the following:

If $D \subseteq C \subseteq B \subseteq A$, then $d(A, D) \geq d(A, C) \geq d(B, C)$, $d(A, D) \geq d(B, D) \geq d(B, C) \rightarrow d(A, D) \geq d(B, C)$.

If $D \subset C \subset B \subset A$, then $d(A, D)>d(A, C)>d(B, C)$, $d(A, D)>d(B, D)>d(B, C) \rightarrow d(A, D)>d(B, C)$.

Obviously, for all the distance formulas above, (P1), (P2), and (P3) can be obtained. However, most of them do not satisfy the ranking property $(\mathrm{P} 4)$.

Example 11. Let $A=(0.5,0.4,0.1), B=(0.5,0.5,0), C=$ $(0.4,0.4,0.2)$, and $D=(0.4,0.5,0.1)$. For IFS $A$, we obtain $\mu_{A}(x)=0.5, v_{A}(x)=0.4$, and $\pi_{A}(x)=0.1$. In the following, we denote $\mu_{A}=\mu_{A}(x)=0.5, v_{A}=\nu_{A}(x)=0.4$, and $\pi_{A}=\pi_{A}(x)=0.1$.

According to Definition 3, if $\mu^{-}(x)=\mu^{+}(x)=\mu(x)$, $\nu^{-}(x)=\nu^{+}(x)=\nu(x), \pi^{-}(x)=\pi^{+}(x)=\pi(x)$, then IVIFS is equivalent to IFS. Correspondingly, we can define some IVIFSs as follows, respectively:

$$
\begin{aligned}
& A=\langle x,[0.5,0.5],[0.4,0.4]\rangle, \\
& B=\langle x,[0.5,0.5],[0.5,0.5]\rangle, \\
& C=\langle x,[0.4,0.4],[0.4,0.4]\rangle, \\
& D=\langle x,[0.4,0.4],[0.5,0.5]\rangle .
\end{aligned}
$$

If $X=\left\{x_{1}, x_{2}, \ldots, x_{n}\right\}$ is a universe of discourse, we define

$$
\begin{aligned}
& A=\left\{\left\langle x_{1},[0.5,0.5],[0.4,0.4]\right\rangle,\right. \\
& \left.\ldots,\left\langle x_{n},[0.5,0.5],[0.4,0.4]\right\rangle\right\} ; \\
& B=\left\{\left\langle x_{1},[0.5,0.5],[0.5,0.5]\right\rangle,\right. \\
& \left.\ldots,\left\langle x_{n},[0.5,0.5],[0.5,0.5]\right\rangle\right\} ; \\
& C=\left\{\left\langle x_{1},[0.4,0.4],[0.4,0.4]\right\rangle,\right. \\
& \left.\ldots,\left\langle x_{n},[0.4,0.4],[0.4,0.4]\right\rangle\right\} ; \\
& D=\left\{\left\langle x_{1},[0.4,0.4],[0.5,0.5]\right\rangle,\right. \\
& \left.\ldots,\left\langle x_{n},[0.4,0.4],[0.5,0.5]\right\rangle\right\} \text {. }
\end{aligned}
$$

Thus we have $\mu_{A}(x)=\mu_{B}(x)>\mu_{C}(x)=\mu_{D}(x), \nu_{A}(x)=$ $v_{C}(x)<v_{B}(x)=v_{D}(x)$. According to Definitions 8 and 9 , we have $D \subset C \subset B \subset A$. Therefore, we obtain the necessary condition for distance measure on IFS and IVIFS: $d(A, D)>$ $d(B, C)$.

Obviously, according to $A, B, C$, and $D$, we obtain

$$
\begin{gathered}
\Delta \mu(A, D)=\mu_{A}-\mu_{D}=0.1, \\
\Delta \nu(A, D)=v_{A}-v_{D}=-0.1, \\
\Delta \pi(A, D)=\pi_{A}-\pi_{D}=0, \\
\Delta \mu(B, C)=\mu_{B}-\mu_{C}=0.1, \\
\Delta \nu(B, C)=v_{B}-v_{C}=0.1, \\
\Delta \pi(B, C)=\pi_{B}-\pi_{C}=0.2 .
\end{gathered}
$$

From formulas (8)-(14), we have

$$
\begin{gathered}
d_{\mathrm{Za}}(A, D)=\left(\sum_{i=1}^{n} w_{i} 0.1^{p}\right)^{1 / p}=d_{\mathrm{Za}}(B, C), \\
d_{\mathrm{At}}(A, D)=\left(\sum_{i=1}^{n} w_{i}\left(0.1^{p}+0.1^{p}\right)\right)^{1 / p}=d_{\mathrm{At}}(B, C), \\
d_{\mathrm{S}, \mathrm{K}}(A, D)=\left(\sum_{i=1}^{n} w_{i}\left(0.1^{p}+0.1^{p}\right)\right)^{1 / p} \\
<\left(\sum_{i=1}^{n} w_{i}\left(0.1^{p}+0.1^{p}+0.2^{p}\right)\right)^{1 / p}=d_{\mathrm{S}, \mathrm{K}}(B, C), \\
d_{\mathrm{L}, \mathrm{C}}(A, D)=\left(\sum_{i=1}^{n} w_{i} 0.2^{p}\right)^{1 / p}>0=d_{\mathrm{L}, \mathrm{C}}(B, C),
\end{gathered}
$$




$$
\begin{gathered}
d_{\mathrm{P}, \mathrm{H}}(A, D)=\sum_{i=1}^{n} w_{i} \max (0.1,0.1)=d_{\mathrm{P}, \mathrm{H}}(B, C), \\
d_{\mathrm{W}}(A, D)=\sum_{i=1}^{n} w_{i} \frac{0.1+0.1+2 \max (0.1,0.1)}{4}=d_{\mathrm{W}}(B, C), \\
d_{\mathrm{RB}}(A, D)=+\infty>1=d_{\mathrm{RB}}(B, C) .
\end{gathered}
$$

Only (11) and (14) satisfy property (P4).

Considering (15), we get

$$
\begin{aligned}
d_{D_{k}}(A, D) & =\left(\sum_{i=1}^{n} w_{i}\left(0.1^{p}\right)\right)^{1 / p} \\
& \geq\left(\sum_{i=1}^{n} w_{i}(|1-2 k| 0.1)^{p}\right)^{1 / p} \\
& =d_{D_{k}}(B, C) .
\end{aligned}
$$

If $k \neq 0$ and $k \neq 1$, then we have $d_{D_{k}}(A, D)>d_{D_{k}}(B, C)$; otherwise, we have $d_{D_{k}}(A, D)=d_{D_{k}}(B, C)$.

From (16), we get

$$
\begin{gathered}
d_{F_{k, \lambda}}(A, D)=\left(0.5 \sum_{i=1}^{n} w_{i}\left(0.1^{p}+0.1^{p}\right)\right)^{1 / p}, \\
d_{F_{k, \lambda}}(B, C) \\
=\left(0.5 \sum_{i=1}^{n} w_{i} 0.1^{p}\left(|1-2 k|^{p}+|1-2 \lambda|^{p}+|1-k-\lambda|^{p}\right)\right)^{1 / p}, \\
k+\lambda \leq 1 .
\end{gathered}
$$

If $p=1$, then we have $k+\lambda<1 / 2 \rightarrow d_{D_{k}}(A, D)<d_{D_{k}}(B, C)$; $k+\lambda \geq 1 / 2 \rightarrow d_{D_{k}}(A, D) \geq d_{D_{k}}(B, C)$.

From (17), we get

$$
\begin{aligned}
d_{G_{k, \lambda}} & (A, D) \\
& =\left(0.5 \sum_{i=1}^{n} w_{i}\left(|0.1 k|^{p}+|0.1 \lambda|^{p}+|0.1(k-\lambda)|^{p}\right)\right)^{1 / p} \\
& \leq\left(0.5 \sum_{i=1}^{n} w_{i}\left(|0.1 k|^{p}+|0.1 \lambda|^{p}+|0.1(k+\lambda)|^{p}\right)\right)^{1 / p} \\
& =d_{G_{k, \lambda}}(B, C) .
\end{aligned}
$$

Obviously, we get $d_{D_{k}}(A, D) \leq d_{D_{k}}(B, C)$ for each $k \geq 0$, for each $\lambda \geq 0$, and for each $P>0$.
For (18), we have

$$
\begin{aligned}
& d_{H_{k, \lambda}}(A, D) \\
& =\left(0.5 \sum_{i=1}^{n} w_{i}\left(|0.1 k|^{p}+|0.1|^{p}+|0.1(1-k)|^{p}\right)\right)^{1 / p}, \\
& d_{H_{k, \lambda}}(B, C) \\
& =\left(0 . 5 \sum _ { i = 1 } ^ { n } w _ { i } \left(|0.1 k|^{p}+|0.1(1-2 \lambda)|^{p}\right.\right. \\
& \left.\left.+|0.1(1+k-2 \lambda)|^{p}\right)\right)^{1 / p} .
\end{aligned}
$$

If $p=1$ and $k+\lambda \leq 1$, then we have $0 \leq \lambda<1 / 3,2 \lambda<k \rightarrow$ $d_{H_{k, \lambda}}(A, D)<d_{H_{k, \lambda}}(B, C)$; otherwise, $d_{D_{k}}(A, D) \geq d_{D_{k}}(B, C)$.

For example, let $p=1, \lambda=0.2$, and $k=0.5$, and then $d_{H_{k, \lambda}}(A, D)=0.5 \sum_{i=1}^{n} w_{i} 0.2<0.5 \sum_{i=1}^{n} w_{i} 0.22=d_{H_{k, \lambda}}(B, C)$.

Similar to the result from (18), for (20), we have

$$
\begin{gathered}
d_{J_{k, \lambda}}(A, D) \\
=\left(0.5 \sum_{i=1}^{n} w_{i}\left(|0.1|^{p}+|0.1 \lambda|^{p}+|0.1(1-\lambda)|^{p}\right)\right)^{1 / p}, \\
d_{J_{k, \lambda}}(B, C) \\
=\left(0 . 5 \sum _ { i = 1 } ^ { n } w _ { i } \left(|0.1(1-2 k)|^{p}+|0.1 \lambda|^{p}\right.\right. \\
\left.\left.+|0.1(1+\lambda-2 k)|^{p}\right)\right)^{1 / p} .
\end{gathered}
$$

If $p=1$ and $k+\lambda \leq 1$, then we have $0 \leq k<1 / 3,2 k<\lambda \rightarrow$ $d_{H_{k, \lambda}}(A, D)<d_{H_{k, \lambda}}(B, C)$; otherwise, $d_{D_{k}}(A, D) \geq d_{D_{k}}(B, C)$.

For example, let $p=1, k=0.2$, and $\lambda=0.5$, and then $d_{H_{k, \lambda}}(A, D)=0.5 \sum_{i=1}^{n} w_{i} 0.2<0.5 \sum_{i=1}^{n} w_{i} 0.22=d_{H_{k, \lambda}}(B, C)$. For (19), we obtain

$$
\begin{array}{r}
d_{H_{k, \lambda}^{*}}(A, D) \\
=\left(0 . 5 \sum _ { i = 1 } ^ { n } w _ { i } \left(|0.1 k|^{p}+|0.1(1+k \lambda-\lambda)|^{p}\right.\right. \\
\left.\left.+|0.1(1-\lambda)(1-k)|^{p}\right)\right)^{1 / p}, \\
d_{H_{k, \lambda}^{*}}(B, C) \\
=\left(0 . 5 \sum _ { i = 1 } ^ { n } w _ { i } \left(|0.1 k|^{p}+|0.1(1-k \lambda-\lambda)|^{p}\right.\right. \\
\left.\left.+|0.1(1-\lambda)(1+k)|^{p}\right)\right)^{1 / p}, \\
k+\lambda \leq 1 .
\end{array}
$$


If $p=1$ and $k+\lambda \leq 1$, then we have $0 \leq \lambda<1 / 2 \rightarrow$ $d_{H_{k, \lambda}}(A, D)<d_{H_{k, \lambda}}(B, C)$; otherwise, $d_{D_{k}}(A, D) \geq d_{D_{k}}(B, C)$.

Similarly, we have the same results for (21), which will be as follows:

if $p=1$ and $k+\lambda \leq 1$, then $0 \leq k<1 / 2 \rightarrow$ $d_{H_{k, \lambda}}(A, D)<d_{H_{k, \lambda}}(B, C)$; otherwise, $d_{D_{k}}(A, D) \geq$ $d_{D_{k}}(B, C)$.

From the analysis to the formulas from Xia and $\mathrm{Xu}$ above, only (15) basically meets the requirement of (P4); the others only meet the requirement of (P4) in part. Similarly, most of the conventional distance measures of IVIFS do not satisfy property (P4). Therefore, it is necessary to find a distance measure satisfying the necessary ranking property. Considering the component elements of the distance formula of IFS and IVIFS, we can define some appropriate distance measures of DIVIFS (Figure 2).

According to Definition 7 and Example 11, we have

$$
\begin{aligned}
& \mu_{A}^{-}(x)=0.5, v_{A}^{-}(x)=0.4, \pi_{A}^{+}(x)=0.1, \mu_{A}^{+}(x)=0.5+ \\
& \lambda_{0} \lambda_{1} 0.1, v_{A}^{+}(x)=0.4+\lambda_{0}\left(1-\lambda_{1}\right) 0.1, \pi_{A}^{-}(x)=(1- \\
& \left.\lambda_{0}\right) 0.1, \\
& \mu_{B}^{-}(x)=0.5, v_{B}^{-}(x)=0.5, \pi_{B}^{-}(x)=0, \mu_{B}^{+}(x)=0.5, \\
& v_{B}^{+}(x)=0.5, \pi_{B}^{+}(x)=0, \\
& \mu_{C}^{-}(x)=0.4, v_{C}^{-}(x)=0.4, \pi_{C}^{+}(x)=0.2, \mu_{C}^{+}(x)=0.4+ \\
& \lambda_{0} \lambda_{1} 0.2, v_{C}^{+}(x)=0.4+\lambda_{0}\left(1-\lambda_{1}\right) 0.2, \pi_{C}^{-}(x)=(1- \\
& \left.\lambda_{0}\right) 0.2, \\
& \mu_{D}^{-}(x)=0.4, v_{D}^{-}(x)=0.5, \pi_{D}^{+}(x)=0.1, \mu_{D}^{+}(x)=0.4+ \\
& \lambda_{0} \lambda_{1} 0.1, v_{D}^{+}(x)=0.5+\lambda_{0}\left(1-\lambda_{1}\right) 0.1, \pi_{D}^{-}(x)=(1- \\
& \left.\lambda_{0}\right) 0.1 .
\end{aligned}
$$

Let $\mu^{-}=\mu^{-}(x), \mu^{+}=\mu^{+}(x), v^{-}=\nu^{-}(x), v^{+}=\nu^{+}(x), \pi^{-}=$ $\pi^{-}(x), \pi^{+}=\pi^{+}(x)$, and we obtain

(1) $\Delta \pi^{-}(A, D)=\pi_{A}^{-}-\pi_{D}^{-}=0, \Delta \pi^{-}(B, C)=\pi_{B}^{-}-\pi_{C}^{-}=$ $-\left(1-\lambda_{0}\right) 0.2 \rightarrow\left|\Delta \pi^{-}(A, D)\right| \leq\left|\Delta \pi^{-}(B, C)\right| ;$

(2) $\Delta \pi^{+}(A, D)=\pi_{A}^{+}-\pi_{D}^{+}=0<\pi_{B}^{+}-\pi_{C}^{+}=\Delta \pi^{+}(B, C)=$ $0.2 \rightarrow\left|\Delta \pi^{+}(A, D)\right|<\left|\Delta \pi^{+}(B, C)\right|$;

(3) $\Delta \mu^{-}(A, D)=\mu_{A}^{-}-\mu_{D}^{-}=0.1=\mu_{B}^{-}-\mu_{C}^{-}=\Delta \mu^{-}(B, C) \rightarrow$ $\left|\Delta \mu^{-}(A, D)\right|=\left|\Delta \mu^{-}(B, C)\right| ;$

(4) $\Delta v^{-}(A, D)=v_{A}^{-}-v_{D}^{-}=-0.1=-1 \times\left(v_{B}^{-}-v_{C}^{-}\right)=$ $-\Delta \nu^{-}(B, C) \rightarrow\left|\Delta \nu^{-}(A, D)\right|=\left|\Delta \nu^{-}(B, C)\right| ;$

(5) $\Delta \mu^{-}(A, D)-\Delta v^{-}(A, D)=0.2>0=$ $\Delta \mu^{-}(B, C)-\Delta \nu^{-}(B, C) \rightarrow\left|\Delta \mu^{-}(A, D)-\Delta \nu^{-}(A, D)\right|>$ $\left|\Delta \mu^{-}(B, C)-\Delta v^{-}(B, C)\right|$;

(6) $\Delta \mu^{+}(A, D)=\mu_{A}^{+}-\mu_{D}^{+}=0.1, \Delta \mu^{+}(B, C)=\mu_{B}^{+}-\mu_{C}^{+}=$ $0.1\left(1-2 \lambda_{0} \lambda_{1}\right) \rightarrow\left|\Delta \mu^{+}(A, D)\right| \geq\left|\Delta \mu^{+}(B, C)\right| ;$

(7) $\Delta v^{+}(A, D)=v_{A}^{+}-v_{D}^{+}=-0.1, \Delta v^{+}(B, C)=v_{B}^{+}-v_{C}^{+}=$ $0.1\left(1-2 \lambda_{0}\left(1-\lambda_{1}\right)\right) \rightarrow\left|\Delta \mu^{+}(A, D)\right| \geq\left|\Delta \mu^{+}(B, C)\right| ;$

(8) $\Delta \mu^{+}(A, D)-\Delta v^{+}(A, D)=0.2, \Delta \mu^{+}(B, C)-\Delta v^{+}(B, C)=$ $0.2 \lambda_{0}\left(1-2 \lambda_{1}\right), \rightarrow\left|\Delta \mu^{+}(A, D)-\Delta \nu^{+}(A, D)\right| \geq$ $\left|\Delta \mu^{+}(B, C)-\Delta \nu^{+}(B, C)\right|$.

Considering the necessary condition (P4), we draw a conclusion as follows. $\Delta \pi^{+}$does not meet the requirement of the ranking property, and if $\lambda_{0} \neq 1, \Delta \pi^{-}$does not satisfy (P4) too. $\Delta \mathcal{u}^{-}-\Delta v^{-}$meets the necessary condition (P4), and $\Delta \mu^{+}$, $\Delta v^{+}$, and $\Delta \mu^{+}-\Delta v^{+}$do so if $\lambda_{k} \in(0,1)(k=1,2)$. All the calculated results are shown in (32) and (33):

$$
\begin{gathered}
\left|\Delta \pi^{-}(A, D)\right| \leq\left|\Delta \pi^{-}(B, C)\right| ; \\
\left|\Delta \pi^{-}(A, D)\right|=\left|\Delta \pi^{-}(B, C)\right| \Longleftrightarrow \lambda_{0}=1 ; \\
\left|\Delta \pi^{+}(A, D)\right|=\left|\Delta \pi^{+}(B, C)\right| ; \\
\left|\Delta \mu^{-}(A, D)\right|=\left|\Delta \mu^{-}(B, C)\right| ; \\
\left|\Delta v^{-}(A, D)\right|=\left|\Delta v^{-}(B, C)\right| ; \\
\left|\Delta \mu^{-}(A, D)-\Delta v^{-}(A, D)\right| \\
>\left|\Delta \mu^{-}(B, C)-\Delta v^{-}(B, C)\right| ; \\
\left|\Delta \mu^{+}(A, D)\right| \geq\left|\Delta \mu^{+}(B, C)\right| ;
\end{gathered}
$$

$\left|\Delta \mu^{+}(A, D)\right|$

$=\left|\Delta \mu^{+}(B, C)\right|$

$\Longleftrightarrow \lambda_{0}=0, \quad$ or $\quad \lambda_{1}=0, \quad$ or $\quad \lambda_{0}=\lambda_{1}=1$;

$\left|\Delta \nu^{+}(A, D)\right| \geq\left|\Delta \nu^{+}(B, C)\right| ;$

$$
\begin{aligned}
& \left|\Delta \nu^{+}(A, D)\right| \\
& \quad=\left|\Delta \nu^{+}(B, C)\right| \\
& \quad \Longleftrightarrow \lambda_{0}=0, \quad \text { or } \quad \lambda_{1}=1, \quad \text { or } \lambda_{0}=1, \lambda_{1}=0 ;
\end{aligned}
$$

$$
\begin{gathered}
\left|\Delta \mu^{+}(A, D)-\Delta \nu^{+}(A, D)\right| \\
\geq\left|\Delta \mu^{+}(B, C)-\Delta \nu^{+}(B, C)\right| ; \\
\left|\Delta \mu^{+}(A, D)-\Delta \nu^{+}(A, D)\right| \\
=\left|\Delta \mu^{+}(B, C)-\Delta \nu^{+}(B, C)\right| \\
\Longleftrightarrow \lambda_{0}=1, \lambda_{1}=0, \quad \text { or } \quad \lambda_{0}=1, \lambda_{1}=1 .
\end{gathered}
$$

In all, in order to meet the needs of necessary condition (P4), we conclude that the distance measure of DIVIFS should be composed of $\Delta \mu^{-}-\Delta v^{-}, \Delta \mu^{+}, \Delta v^{+}$, and $\Delta \mu^{+}-\Delta v^{+}$ and that $\Delta \mu^{-}$and $\Delta \nu^{-}$can be involved in the distance formula too. However, considering the contradiction between $\Delta \pi$ and (P4), $\Delta \pi^{+}$and $\Delta \pi^{-}$are not suitable for new distance measures.

In Xu's papers $[16,17,19,20]$, many weighted similarity measures are proposed according to standardized distance measures based on the IFS theory and the IVIFS theory. And $\mathrm{Xu}$ presented them by defining the appropriate weights of membership function, non-membership function, and hesitancy function. According to $\mathrm{Xu}$, we can also define 


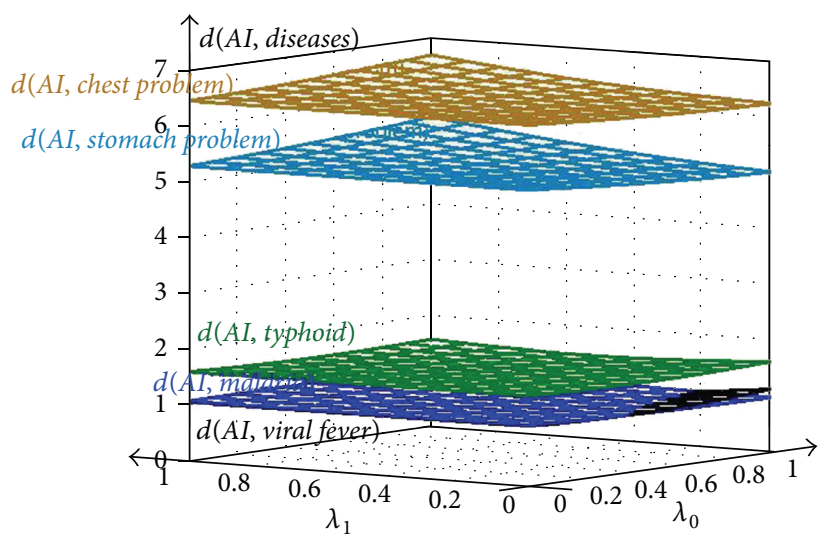

(a)

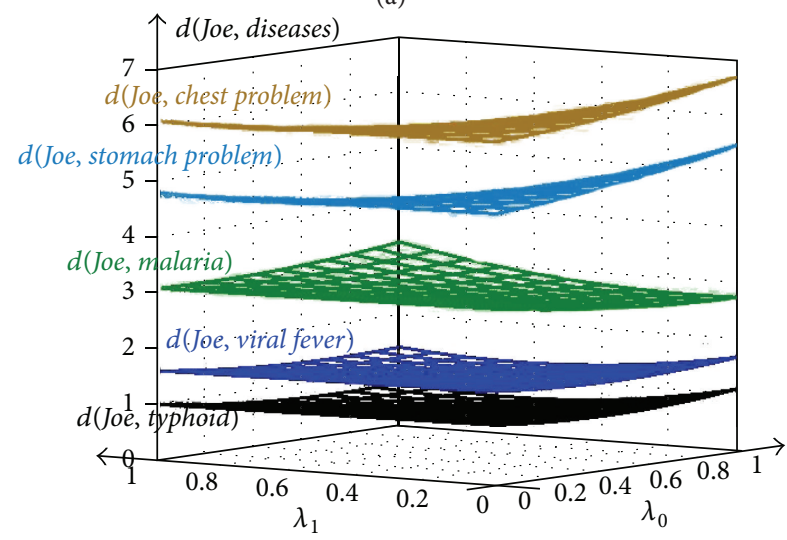

(c)

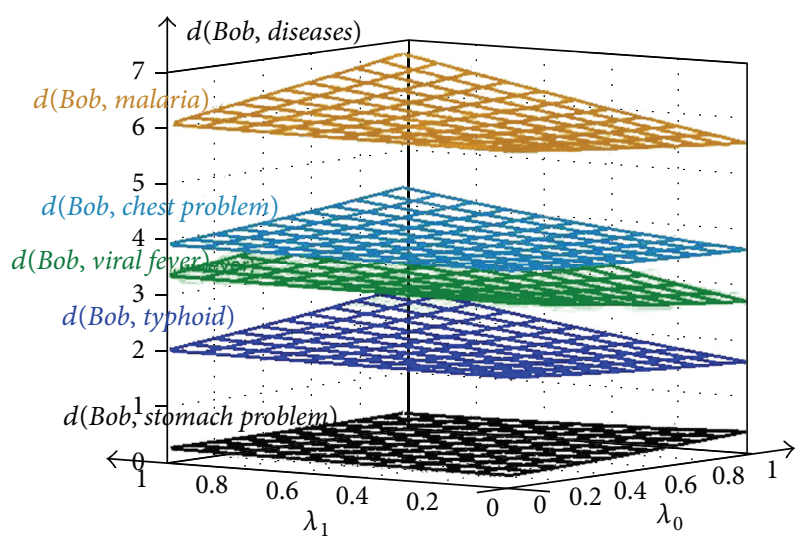

(b)

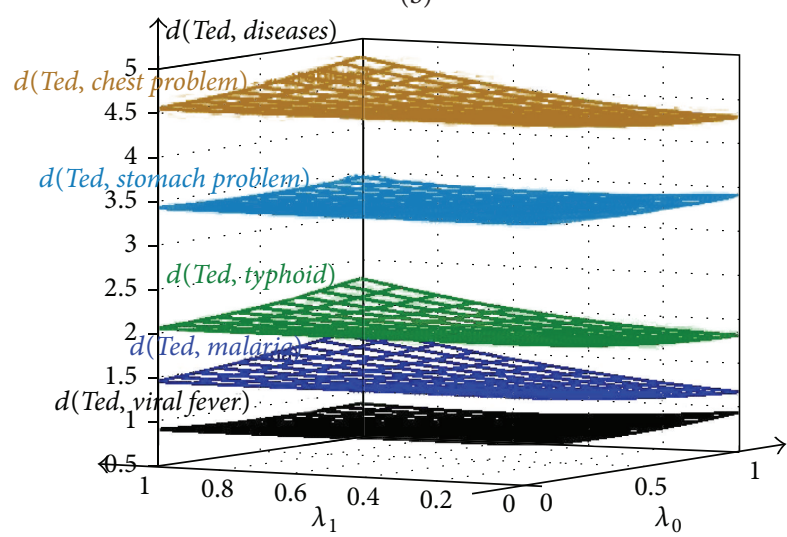

(d)

FIGURE 2: Distance between each patient and each disease on DIVIFS.

the corresponding weighted distance measures on IVIFS as follows:

$$
\begin{aligned}
d(A, B) & \\
=\left\{\sum_{i=1}^{n} w_{i}\right. & {\left[C_{1}\left|\Delta \mu^{-}\left(A\left(x_{i}\right), B\left(x_{i}\right)\right)\right|^{p}\right.} \\
& +C_{2}\left|\Delta \mu^{+}\left(A\left(x_{i}\right), B\left(x_{i}\right)\right)\right|^{p} \\
& +C_{3}\left|\Delta \nu^{-}\left(A\left(x_{i}\right), B\left(x_{i}\right)\right)\right|^{p} \\
& +C_{4}\left|\Delta \nu^{+}\left(A\left(x_{i}\right), B\left(x_{i}\right)\right)\right|^{p} \\
& +\mid C_{5} \Delta \mu^{-}\left(A\left(x_{i}\right), B\left(x_{i}\right)\right) \\
& -\left.C_{7} \Delta \nu^{-}\left(A\left(x_{i}\right), B\left(x_{i}\right)\right)\right|^{p} \\
& +\mid C_{6} \Delta \mu^{+}\left(A\left(x_{i}\right), B\left(x_{i}\right)\right) \\
& \left.\left.-\left.C_{8} \Delta \nu^{+}\left(A\left(x_{i}\right), B\left(x_{i}\right)\right)\right|^{p}\right]\right\} ; \\
& \sum_{i=1}^{n} w_{i}=1, \quad w_{i} \geq 0 ; \\
C_{j} \geq & 0, \quad j=1,2,3,4,5,6,7,8 .
\end{aligned}
$$

It is easy to prove that (11) is equivalent to a special case of (15) when $k=0.5$. And (15) is also a special case of (34) when $C_{1}=C_{2}=C_{3}=C_{4}=C_{6}=C_{8}=0$ and $C_{5}=C_{7}=1$. To simplifiy the formula, let $C_{5}=C_{7}$ and $C_{6}=C_{8}$. Assume that $A$ and $B$ are two DIVIFS, and we have

$$
\begin{aligned}
& d(A, B) \\
& =\left\{\sum _ { i = 1 } ^ { n } w _ { i } \left[C_{1}\left|\mu_{A}^{-}\left(x_{i}\right)-\mu_{B}^{-}\left(x_{i}\right)\right|^{p}+C_{2}\left|\nu_{A}^{-}\left(x_{i}\right)-\nu_{B}^{-}\left(x_{i}\right)\right|^{p}\right.\right. \\
& +C_{3} \mid \mu_{A}^{-}\left(x_{i}\right)-\mu_{B}^{-}\left(x_{i}\right) \\
& +\left.\lambda_{0} \lambda_{1}\left(\pi_{A}^{+}\left(x_{i}\right)-\pi_{B}^{+}\left(x_{i}\right)\right)\right|^{p} \\
& +C_{4} \mid \nu_{A}^{-}\left(x_{i}\right)-v_{B}^{-}\left(x_{i}\right) \\
& +\left.\lambda_{0}\left(1-\lambda_{1}\right)\left(\pi_{A}^{+}\left(x_{i}\right)-\pi_{B}^{+}\left(x_{i}\right)\right)\right|^{p} \\
& +C_{5}\left|\left(\mu_{A}^{-}\left(x_{i}\right)-\mu_{B}^{-}\left(x_{i}\right)\right)-\left(v_{A}^{-}\left(x_{i}\right)-\nu_{B}^{-}\left(x_{i}\right)\right)\right|^{p} \\
& +C_{6} \mid\left(\mu_{A}^{-}\left(x_{i}\right)-\mu_{B}^{-}\left(x_{i}\right)\right)-\left(\nu_{A}^{-}\left(x_{i}\right)-\nu_{B}^{-}\left(x_{i}\right)\right) \\
& \left.\left.+\left.\lambda_{0}\left(2 \lambda_{1}-1\right)\left(\pi_{A}^{+}\left(x_{i}\right)-\pi_{B}^{+}\left(x_{i}\right)\right)\right|^{p}\right]\right\}^{1 / p}, \\
& \sum_{i=1}^{n} w_{i}=1, \quad w_{i} \geq 0 ; \quad C_{j} \geq 0, \quad j=1,2,3,4,5,6 .
\end{aligned}
$$


Comparing (15)-(21) with (34)-(35), we conclude that Xu's methods are different from the DIVIFS method. Xu defined the measures by constructing the weights of membership function, non-membership function, and hesitancy function, respectively. However, we concentrate on the detachment of hesitancy function and present the DIVIFS method. If we use Xu's methods solely, we can define the weights of certainty and uncertainty, but we cannot analyze the variation of the absent party. Moreover, in Xu's research, and even in all the conventional research, there is no research on the ranking condition (P4). Therefore, the distance measures on DIVIFS above are an important supplement to Xu's methods and the others'.

In order to simplify the calculation, we define the following distance measure:

$$
\begin{gathered}
d(A, B) \\
=\sum_{i=1}^{n} w_{i}\left[C_{1}\left|\mu_{A}^{-}\left(x_{i}\right)-\mu_{B}^{-}\left(x_{i}\right)\right|^{p}+C_{2}\left|\nu_{A}^{-}\left(x_{i}\right)-v_{B}^{-}\left(x_{i}\right)\right|^{p}\right. \\
+C_{3} \mid \mu_{A}^{-}\left(x_{i}\right)-\mu_{B}^{-}\left(x_{i}\right) \\
+\left.\lambda_{0} \lambda_{1}\left(\pi_{A}^{+}\left(x_{i}\right)-\pi_{B}^{+}\left(x_{i}\right)\right)\right|^{p} \\
+C_{4} \mid \nu_{A}^{-}\left(x_{i}\right)-\nu_{B}^{-}\left(x_{i}\right) \\
+\left.\lambda_{0}\left(1-\lambda_{1}\right)\left(\pi_{A}^{+}\left(x_{i}\right)-\pi_{B}^{+}\left(x_{i}\right)\right)\right|^{p} \\
+C_{5}\left|\left(\mu_{A}^{-}\left(x_{i}\right)-\mu_{B}^{-}\left(x_{i}\right)\right)-\left(v_{A}^{-}\left(x_{i}\right)-v_{B}^{-}\left(x_{i}\right)\right)\right|^{p} \\
+C_{6} \mid\left(\mu_{A}^{-}\left(x_{i}\right)-\mu_{B}^{-}\left(x_{i}\right)\right)-\left(v_{A}^{-}\left(x_{i}\right)-v_{B}^{-}\left(x_{i}\right)\right) \\
\left.\quad+\left.\lambda_{0}\left(2 \lambda_{1}-1\right)\left(\pi_{A}^{+}\left(x_{i}\right)-\pi_{B}^{+}\left(x_{i}\right)\right)\right|^{p}\right], \\
\sum_{i=1}^{n} w_{i}=1, \quad w_{i} \geq 0 ; \\
C_{j} \geq 0, \quad j=1,2,3,4,5,6 .
\end{gathered}
$$

If $p=1$, the distance measure is the Hamming distance; if $p=2$, it is the Euclidean distance. In this paper, the Euclidean distance on DIVIFS will be used in the simulation of pattern recognition:

$$
\begin{aligned}
& d(A, B) \\
& =\sum_{i=1}^{n}\left\{\left[\mu_{A}^{-}\left(x_{i}\right)-\mu_{B}^{-}\left(x_{i}\right)\right]^{2}+\left[\nu_{A}^{-}\left(x_{i}\right)-v_{B}^{-}\left(x_{i}\right)\right]^{2}\right. \\
& +\left[\mu_{A}^{-}\left(x_{i}\right)-\mu_{B}^{-}\left(x_{i}\right)+\lambda_{0} \lambda_{1}\left(\pi_{A}^{+}\left(x_{i}\right)-\pi_{B}^{+}\left(x_{i}\right)\right)\right]^{2} \\
& +\left[\nu_{A}^{-}\left(x_{i}\right)-\nu_{B}^{-}\left(x_{i}\right)\right. \\
& \left.\left.+\lambda_{0}\left(1-\lambda_{1}\right)\left(\pi_{A}^{+}\left(x_{i}\right)-\pi_{B}^{+}\left(x_{i}\right)\right)\right]^{2}\right\}
\end{aligned}
$$

$d(A, B)$

$$
\begin{gathered}
=\sum_{i=1}^{n}\left\{\left[\left(\mu_{A}^{-}\left(x_{i}\right)-\mu_{B}^{-}\left(x_{i}\right)\right)-\left(v_{A}^{-}\left(x_{i}\right)-v_{B}^{-}\left(x_{i}\right)\right)\right]^{2}\right. \\
+\left[\left(\mu_{A}^{-}\left(x_{i}\right)-\mu_{B}^{-}\left(x_{i}\right)\right)-\left(v_{A}^{-}\left(x_{i}\right)-v_{B}^{-}\left(x_{i}\right)\right)\right. \\
\left.\left.+\lambda_{0}\left(2 \lambda_{1}-1\right)\left(\pi_{A}^{+}\left(x_{i}\right)-\pi_{B}^{+}\left(x_{i}\right)\right)\right]^{2}\right\},
\end{gathered}
$$

$d(A, B)$

$$
\begin{aligned}
=\sum_{i=1}^{n}\left\{\left[\mu_{A}^{-}\left(x_{i}\right)-\mu_{B}^{-}\left(x_{i}\right)\right]^{2}+\left[\nu_{A}^{-}\left(x_{i}\right)-v_{B}^{-}\left(x_{i}\right)\right]^{2}\right. \\
+\quad\left[\mu_{A}^{-}\left(x_{i}\right)-\mu_{B}^{-}\left(x_{i}\right)+\lambda_{0} \lambda_{1}\left(\pi_{A}^{+}\left(x_{i}\right)-\pi_{B}^{+}\left(x_{i}\right)\right)\right]^{2} \\
+\quad\left[\nu_{A}^{-}\left(x_{i}\right)-v_{B}^{-}\left(x_{i}\right)\right. \\
\left.\quad+\lambda_{0}\left(1-\lambda_{1}\right)\left(\pi_{A}^{+}\left(x_{i}\right)-\pi_{B}^{+}\left(x_{i}\right)\right)\right]^{2} \\
+\quad\left[\left(\mu_{A}^{-}\left(x_{i}\right)-\mu_{B}^{-}\left(x_{i}\right)\right)-\left(v_{A}^{-}\left(x_{i}\right)-v_{B}^{-}\left(x_{i}\right)\right)\right]^{2} \\
+\left[\left(\mu_{A}^{-}\left(x_{i}\right)-\mu_{B}^{-}\left(x_{i}\right)\right)-\left(v_{A}^{-}\left(x_{i}\right)-v_{B}^{-}\left(x_{i}\right)\right)\right. \\
\left.\left.+\lambda_{0}\left(2 \lambda_{1}-1\right)\left(\pi_{A}^{+}\left(x_{i}\right)-\pi_{B}^{+}\left(x_{i}\right)\right)\right]^{2}\right\} .
\end{aligned}
$$

\section{Application to Medical Diagnosis Based on Pattern Recognition}

To make a proper diagnosis $D$ for a patient with given values of symptoms $S$, a medical knowledge base is necessary that involves elements described in terms of IFS. We adopt the same data as those in $[8,9]$ : the set of disease diagnoses is $D=\{$ Viral fever, Malaria, Typhoid, Stomach problem, Chest problem\}, and the set of symptoms is a universe of discourse $S=$ \{temperature, headache, stomach pain, cough, chest pain\}. The data are given in Table 1, where each symptom is described as follows: membership function $\mu_{A}(x)$, nonmembership function $v_{A}(x)$, and hesitancy function $\pi_{A}(x)$. For example, for malaria the temperature is high $\left(\mu_{A}(x)=\right.$ $\left.0.7, \nu_{A}(x)=0, \pi_{A}(x)=0.3\right)$, while for chest problem the temperature is low $\left(\mu_{A}(x)=0.1, \nu_{A}(x)=0.8, \pi_{A}(x)=0.1\right)$, and so forth.

The set of patients is $P=\{A I, B o b, J o e, T e d\}$. The symptoms are given in Table 2, where we need all three parameters $\left(\mu_{A}(x), \nu_{A}(x), \pi_{A}(x)\right)$ to describe each symptom (see $[8,9])$ as before. We seek a diagnosis for each patient $p_{j}$, $j=1,2,3,4$. In [8, 9], Szmidt and Kacprzyk [8, 9] proposed to solve the problem in the following way: (1) to calculate a distance from a set of symptoms $s_{i}(i=1,2,3,4,5)$ (using the normalized Hamming distance, the Euclidean distance, and ratio-based measure of similarity) between each patient $p_{j}$ $(j=1,2,3,4$, Table 2$)$ and each diagnosis $d_{k},(k=1,2,3,4,5$, Table 1); (2) to determine that the shortest distance should be a proper diagnosis.

For (10), the normalized Hamming distance for all symptoms of patient $j$ th from diagnosis $k$ th is (40), and 
TABLE 1: Symptoms characteristic of the diagnoses under consideration.

\begin{tabular}{|c|c|c|c|c|c|}
\hline $\begin{array}{l}\text { Disease } \\
\text { symptoms }\end{array}$ & Temperature & Headache & Stomach pain & Cough & Chest pain \\
\hline Viral fever & $(0.4,0.0,0.6)$ & $(0.3,0.5,0.2)$ & $(0.1,0.7,0.2)$ & $(0.4,0.3,0.3)$ & $(0.1,0.7,0.2)$ \\
\hline Malaria & $(0.7,0.0,0.3)$ & $(0.2,0.6,0.2)$ & $(0.0,0.9,0.1)$ & $(0.7,0.0,0.3)$ & $(0.1,0.8,0.1)$ \\
\hline Typhoid & $(0.3,0.3,0.4)$ & $(0.6,0.1,0.3)$ & $(0.2,0.7,0.1)$ & $(0.2,0.6,0.2)$ & $(0.1,0.9,0.0)$ \\
\hline Stomach problem & $(0.1,0.7,0.2)$ & $(0.2,0.4,0.4)$ & $(0.8,0.0,0.2)$ & $(0.2,0.7,0.1)$ & $(0.2,0.7,0.1)$ \\
\hline Chest problem & $(0.1,0.8,0.1)$ & $(0.0,0.8,0.2)$ & $(0.2,0.8,0.0)$ & $(0.2,0.8,0.0)$ & $(0.8,0.1,0.1)$ \\
\hline
\end{tabular}

TABLE 2: Symptoms characteristic of the patients under observation.

\begin{tabular}{|c|c|c|c|c|c|}
\hline $\begin{array}{l}\text { Patient } \\
\text { symptoms }\end{array}$ & Temperature & Headache & Stomach pain & Cough & Chest pain \\
\hline$A I$ & $(0.8,0.1,0.1)$ & $(0.6,0.1,0.3)$ & $(0.2,0.8,0.0)$ & $(0.6,0.1,0.3)$ & $(0.1,0.6,0.3)$ \\
\hline Bob & $(0.0,0.8,0.2)$ & $(0.4,0.4,0.2)$ & $(0.6,0.1,0.3)$ & $(0.1,0.7,0.2)$ & $(0.1,0.8,0.1)$ \\
\hline Joe & $(0.8,0.1,0.1)$ & $(0.8,0.1,0.1)$ & $(0.0,0.6,0.4)$ & $(0.2,0.7,0.1)$ & $(0.0,0.5,0.5)$ \\
\hline Ted & $(0.6,0.1,0.3)$ & $(0.5,0.4,0.1)$ & $(0.3,0.4,0.3)$ & $(0.7,0.2,0.1)$ & $(0.3,0.4,0.3)$ \\
\hline
\end{tabular}

the normalized Euclidean distance for all symptoms of patient $j$ th from diagnosis $k$ th is (41). From (14), the ratio-based measure of similarity for all symptoms of patient $j$ th from diagnosis $k$ th is $(42)$ :

$$
\begin{aligned}
& d_{H}\left(p_{j}, d_{k}\right) \\
& =\frac{\sum_{i=1}^{5}\left|\mu_{p_{j}}^{-}\left(s_{i}\right)-\mu_{d_{k}}^{-}\left(s_{i}\right)\right|+\left|v_{p_{j}}^{-}\left(s_{i}\right)-v_{d_{k}}^{-}\left(s_{i}\right)\right|+\left(\pi_{p_{j}}^{+}\left(s_{i}\right)-\pi_{d_{k}}^{+}\left(s_{i}\right)\right)}{10}, \\
& d_{E}\left(p_{j}, d_{k}\right) \\
& =\sqrt{\frac{1}{10} \sum_{i=1}^{5}\left(\left|\mu_{p_{j}}^{-}\left(s_{i}\right)-\mu_{d_{k}}^{-}\left(s_{i}\right)\right|^{2}+\left|v_{p_{j}}^{-}\left(s_{i}\right)-v_{d_{k}}^{-}\left(s_{i}\right)\right|^{2}+\left|\pi_{p_{j}}^{+}\left(s_{i}\right)-\pi_{d_{k}}^{+}\left(s_{i}\right)\right|^{2}\right)} \\
& d_{\mathrm{RB}}\left(p_{j}, d_{k}\right)=\frac{d_{H}\left(p_{j}, d_{k}\right)}{d_{H}\left(p_{j}, d_{k}^{C}\right)} .
\end{aligned}
$$

Szmidt and Kacprzyk describe $d_{k}^{C}$ to be a set $\left(\nu_{A}(x)\right.$, $\left.\mu_{A}(x), \pi_{A}(x)\right)$. For example, for malaria the temperature $d_{k}$ is high $\left(\mu_{A}(x)=0.7, v_{A}(x)=0, \pi_{A}(x)=0.3\right)$, whereas $d_{k}^{C}$ is low $\left(\mu_{A}(x)=0, v_{A}(x)=0.7, \pi_{A}(x)=0.3\right)$, and so on.

From the obtained distance measures and similarity measures, Szmidt and Kacprzyk get the results as Table 3. Three types of distances given in Table 3 are described as follows: the first data is the Hamming distance as in (40), the second is the Euclidean distance as in (41), the third is the ratio-based measure as in (42). For example, $d_{H}(A I$, viral fever), $d_{E}(A I$, viral fever $)$, and $d_{\mathrm{RB}}(A I$, viral fever) are 0.28 , 0.29 , and 0.75 respectively. Similarly, we can get the other results. Obviously, Bob suffers from stomach problem and Joe from typhoid based on (40)-(42) above. AI suffers from Malaria and Ted from Viral fever calculated by the Hamming distance and the Euclidean distance, while $A I$ suffers from Viral fever and Ted from Malaria calculated by the ratio-based measure.
Let $p=5$ and $w_{i}=1 / n$ for each $i$, and $k$ and $\lambda$ are constants. Calculated by (15)-(21), Table 4 can be obtained.

From Table 4, we conclude that Bob suffers from stomach problem, Joe from typhoid, Ted from viral fever, and AI from viral fever according to most of Xu's methods. But on the basis of $D_{J 0.1,0.6}, A I$ can be diagnosed as a malaria sufferer. Using Xu's methods of IVIFS from [20], we can get the same results as Table 4 . The results above are also the same as those from [35] by Wei et al.

Let $\mu_{A}^{-}(x)=\mu_{A}(x), \nu_{A}^{-}(x)=\nu_{A}(x)$, and $\pi_{A}^{+}(x)=\pi_{A}(x)$. Using (37), we will get Table 5 .

The distances given in Table 5 are described as follows: for example, from the first data of viral fever and malaria, we can describe the distance measure between $A I$ and viral fever, and the distance measure between $A I$ and malaria from (37), respectively. Similarly, we can get all the results (43), and obtain Table 5 from all (43):

$$
\begin{aligned}
d(\text { AI, Viral fever })= & 1.06-0.24 \lambda_{0}-0.14 \lambda_{0} \lambda_{1} \\
& +0.31 \lambda_{0}^{2}-0.62 \lambda_{0}^{2} \lambda_{1}\left(1-\lambda_{1}\right), \\
d(\text { AI, Malaria })= & 1.08-0.2 \lambda_{0}+0.2 \lambda_{0} \lambda_{1} \\
& +0.1 \lambda_{0}^{2}-0.2 \lambda_{0}^{2} \lambda_{1}\left(1-\lambda_{1}\right), \\
d(\text { AI, Typhoid })= & 1.6-0.18 \lambda_{0}-0.04 \lambda_{0} \lambda_{1} \\
& +0.2 \lambda_{0}^{2}-0.4 \lambda_{0}^{2} \lambda_{1}\left(1-\lambda_{1}\right), \\
d(\text { AI, Stomach problem })= & 5.28-0.42 \lambda_{0}+0.56 \lambda_{0} \lambda_{1} \\
& +0.14 \lambda_{0}^{2}-0.28 \lambda_{0}^{2} \lambda_{1}\left(1-\lambda_{1}\right), \\
& 6.44-0.36 \lambda_{0}+0.44 \lambda_{0} \lambda_{1} \\
& +0.14 \lambda_{0}^{2}-0.28 \lambda_{0}^{2} \lambda_{1}\left(1-\lambda_{1}\right),
\end{aligned}
$$


TABLE 3: Distance description between each patient and each disease based on IFS.

\begin{tabular}{lccccc}
\hline Patient/disease & Viral fever & Malaria & Typhoid & Stomach problem & Chest problem \\
\hline AI & $(0.28,0.29, \mathbf{0 . 7 5})$ & $(\mathbf{0 . 2 4}, \mathbf{0 . 2 5}, 1.19)$ & $(0.28,0.32,1.31)$ & $(0.54,0.53,3.27)$ & $(0.56,0.58, \infty)$ \\
Bob & $(0.40,0.43,2.1)$ & $(0.50,0.56,3.73)$ & $(0.31,0.33,1.10)$ & $(\mathbf{0 . 1 4}, \mathbf{0 . 1 4}, \mathbf{0 . 3 5})$ & $(0.42,0.46, \infty)$ \\
Joe & $(0.38,0.36,0.87)$ & $(0.44,0.41,1.52)$ & $(\mathbf{0 . 3 2}, \mathbf{0 . 3 2}, \mathbf{0 . 4 6 )}$ & $(0.50,0.52,2.61)$ & $(0.55,0.57, \infty)$ \\
Ted & $(\mathbf{0 . 2 8}, \mathbf{0 . 2 5}, 0.95)$ & $(0.30,0.29, \mathbf{0 . 7 7})$ & $(0.38,0.35,1.67)$ & $(0.44,0.43, \infty)$ & $(0.54,0.50,2.56)$ \\
\hline
\end{tabular}

$$
\begin{aligned}
d(\text { Bob }, \text { Viral fever })= & 3.38-0.86 \lambda_{0}+1.34 \lambda_{0} \lambda_{1} \\
& +0.19 \lambda_{0}^{2}-0.38 \lambda_{0}^{2} \lambda_{1}\left(1-\lambda_{1}\right), \\
d(\text { Bob, Malaria })= & 6.12-0.62 \lambda_{0}+1.12 \lambda_{0} \lambda_{1} \\
& +0.06 \lambda_{0}^{2}-0.12 \lambda_{0}^{2} \lambda_{1}\left(1-\lambda_{1}\right), \\
d(\text { Bob }, \text { Typhoid })= & 2.04-0.52 \lambda_{0}+0.84 \lambda_{0} \lambda_{1} \\
& +0.1 \lambda_{0}^{2}-0.2 \lambda_{0}^{2} \lambda_{1}\left(1-\lambda_{1}\right),
\end{aligned}
$$$$
d(\text { Bob }, \text { Stomach problem })=0.28+0.02 \lambda_{0}-0.16 \lambda_{0} \lambda_{1}
$$$$
+0.06 \lambda_{0}^{2}-0.12 \lambda_{0}^{2} \lambda_{1}\left(1-\lambda_{1}\right),
$$$$
d(\text { Bob }, \text { Chest problem })=3.96-0.46 \lambda_{0}+0.64 \lambda_{0} \lambda_{1}
$$$$
+0.14 \lambda_{0}^{2}-0.28 \lambda_{0}^{2} \lambda_{1}\left(1-\lambda_{1}\right),
$$$$
d(\text { Joe, } \text { Viral fever })=1.7-0.34 \lambda_{0}-0.18 \lambda_{0} \lambda_{1}
$$$$
+0.43 \lambda_{0}^{2}-0.86 \lambda_{0}^{2} \lambda_{1}\left(1-\lambda_{1}\right),
$$$$
d(\text { Joe, Malaria })=3.12-0.64 \lambda_{0}+0.6 \lambda_{0} \lambda_{1}
$$$$
+0.34 \lambda_{0}^{2}-0.68 \lambda_{0}^{2} \lambda_{1}\left(1-\lambda_{1}\right),
$$$$
d(\text { Joe }, \text { Typhoid })=1.12-0.36 \lambda_{0}-0.24 \lambda_{0} \lambda_{1}
$$$$
+0.48 \lambda_{0}^{2}-0.96 \lambda_{0}^{2} \lambda_{1}\left(1-\lambda_{1}\right),
$$

$d($ Joe, Stomach problem $)=4.76+0.38 \lambda_{0}-1.36 \lambda_{0} \lambda_{1}$

$$
+0.3 \lambda_{0}^{2}-0.6 \lambda_{0}^{2} \lambda_{1}\left(1-\lambda_{1}\right),
$$

$d($ Joe, Chest problem $)=6+0.28 \lambda_{0}-1.24 \lambda_{0} \lambda_{1}$

$$
+0.34 \lambda_{0}^{2}-0.68 \lambda_{0}^{2} \lambda_{1}\left(1-\lambda_{1}\right)
$$

$d($ Ted, Viral fever $)=0.92-0.12 \lambda_{0}-0.08 \lambda_{0} \lambda_{1}$

$$
+0.16 \lambda_{0}^{2}-0.32 \lambda_{0}^{2} \lambda_{1}\left(1-\lambda_{1}\right)
$$

$d($ Ted, Malaria $)=1.46-0.4 \lambda_{0}+0.54 \lambda_{0} \lambda_{1}$

$$
+0.13 \lambda_{0}^{2}-0.26 \lambda_{0}^{2} \lambda_{1}\left(1-\lambda_{1}\right)
$$

$d($ Ted, Typhoid $)=2.06-0.42 \lambda_{0}+0.46 \lambda_{0} \lambda_{1}$

$$
+0.19 \lambda_{0}^{2}-0.38 \lambda_{0}^{2} \lambda_{1}\left(1-\lambda_{1}\right),
$$

$d($ Ted, Stomach problem $)=3.42-0.16 \lambda_{0}+0.02 \lambda_{0} \lambda_{1}$

$$
+0.15 \lambda_{0}^{2}-0.3 \lambda_{0}^{2} \lambda_{1}\left(1-\lambda_{1}\right),
$$

$$
\begin{aligned}
d(\text { Ted }, \text { Chest problem })= & 4.54-0.44 \lambda_{0}+0.5 \lambda_{0} \lambda_{1} \\
& +0.19 \lambda_{0}^{2}-0.38 \lambda_{0}^{2} \lambda_{1}\left(1-\lambda_{1}\right) .
\end{aligned}
$$

We aim to seek the minimum distance among $d(A I$, disease)s. Therefore, the minimum among all these $d(A I$, disease)s is the pattern we need. From Table 5 , it is clear that if $\lambda_{0}=0, A I, B o b$, Joe, and Ted are identified as having viral fever, stomach problem, typhoid, and viral fever, respectively; if $\lambda_{0}>0, B o b$, Joe, and Ted are also identified as having stomach problem, typhoid, and viral fever, respectively, which is the same as Xu's results in [20] and Wei's results in [35], but $A I$ is different from the above. When $d(A I$, viral fever $)>$ $d(A I$, malaria $), A I$ is identified as malaria; otherwise, $A I$ is viral fever. All in all, we have the following results: if $0.02+$ $0.04 \lambda_{0}+0.34 \lambda_{0} \lambda_{1}-0.21 \lambda_{0}^{2}+0.42 \lambda_{0}^{2} \lambda_{1}\left(1-\lambda_{1}\right)<0$, we can infer that $A I$ suffers from malaria, and on the contrary, $A I$ from viral fever. From Table 5, we conclude that AI suffers from either malaria or viral fever, Bob from stomach problem, Joe from typhoid, and Ted suffers from viral fever. The pattern recognition results are somewhat different from those of the dynamic intuitionistic fuzzy sets (DIFS) method in [26-28], in which we only proposed a DIFS method derived from IFS. However, in this paper, we obtain a series of DIVIFS methods derived from IFS and IVIFS, which are the further study in deep from [26-28].

The experimental results above show that there is a slight difference between the recognition results of DIVIFS and those of IFS. Conventional IFS method is simple, but its recognition results are fixed on the basis of the conventional distance formulas. Therefore, it is difficult to reveal the potential law from all available information when the IFS method is used, whereas the results of the DIVIFS method are flexible, which can be adjusted to sample data with the variables. Furthermore, if the fixed pattern recognition results are different from those of the sample data, the IFS method will fail. However, when using the DIVIFS method, we can meet the requirements of the sample data by adjusting variables to appropriate values. All the results above show that the DIVIFS method is more comprehensive and flexible than the IFS method.

\section{Simulation to Medical Diagnosis}

In the following, we will compare the DIVIFS method with the conventional method in medical diagnosis. We use a sample of 77 women of embryos transferred, which is from Laboratory of Reproductive Medicine, The First Affiliated 


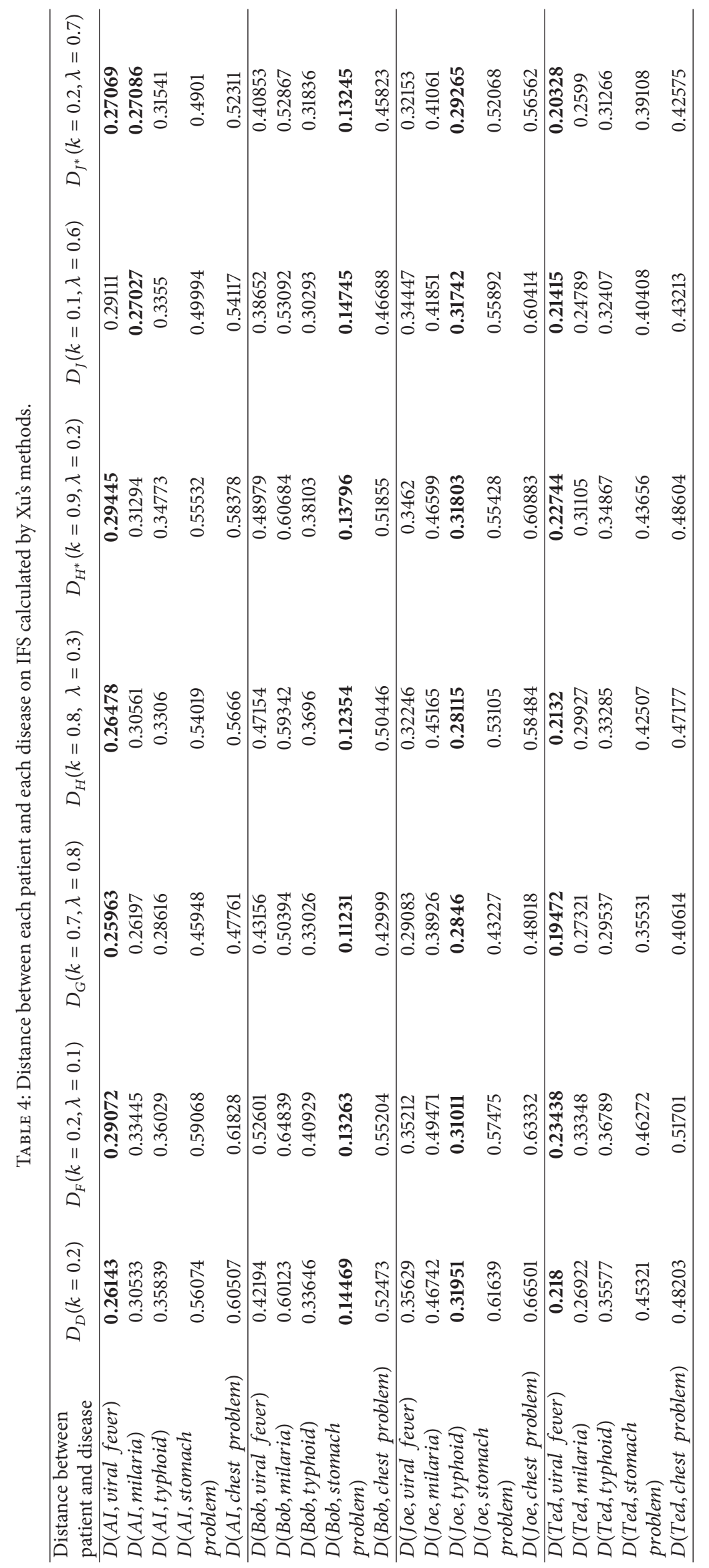




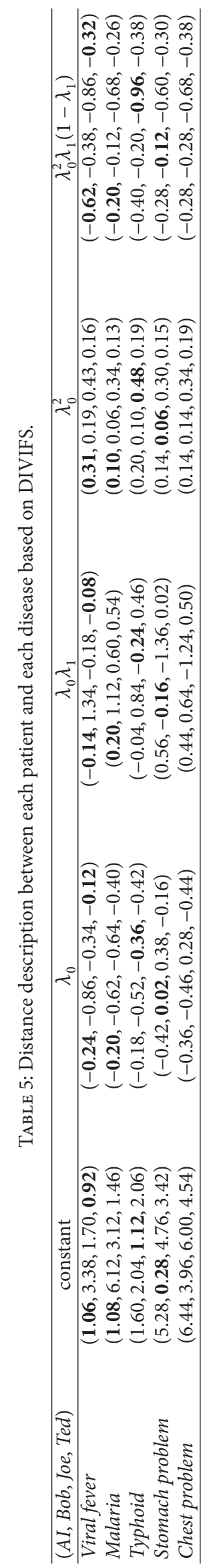


TABLE 6: The optimal results of medical diagnosis based on the distances of IFS and DIVIFS.

\begin{tabular}{lccc}
\hline Equation & Right classification & Wrong classification & Correct recognition rate \\
\hline Equation (15), $k=0.5$ & 57 & 20 & $74.026 \%$ \\
Equation (38), $\lambda_{0} \geq 0.6, \lambda_{1}=0.5$ & 64 & 13 & $83.117 \%$ \\
\hline
\end{tabular}

TABLE 7: Comparison between classification of DIVIFS and existing classification calculated by similarity $(S=1-D$, where $D$ denotes the standardized distance).

\begin{tabular}{|c|c|c|c|c|c|c|}
\hline$i$ & $\begin{array}{c}{\underset{\sim}{A}}_{1}\left(x_{i}\right) \\
\text { Not successful } \\
\text { pregnancy and no } \\
\text { symptoms of OHSS }\end{array}$ & $\begin{array}{c}{\underset{\sim}{A}}_{2}\left(x_{i}\right) \\
\text { Successful } \\
\text { pregnancy and no } \\
\text { symptoms of OHSS }\end{array}$ & $\begin{array}{c}{\underset{\sim}{A}}_{3}\left(x_{i}\right) \\
\text { Not successful } \\
\text { pregnancy and } \\
\text { symptoms of OHSS }\end{array}$ & $\begin{array}{c}{\underset{\sim}{\sim}}_{4}\left(x_{i}\right) \\
\text { Successful } \\
\text { pregnancy and } \\
\text { symptoms of OHSS }\end{array}$ & $\begin{array}{c}\text { DIVIFS } \\
\text { classification } \\
\text { results }\end{array}$ & $\begin{array}{c}D \text { sets } \\
\text { existing } \\
\text { classification }\end{array}$ \\
\hline 1 & 0.572945016 & 0.490904912 & 0.245052718 & 0.258999945 & 1 & 2 \\
\hline 2 & 0.903658468 & 0.866369075 & 0.500009121 & 0.575267057 & 1 & 1 \\
\hline 3 & 0.532136793 & 0.668331297 & 0.402139818 & 0.437292429 & 2 & 2 \\
\hline 4 & 0.569281981 & 0.351763437 & 0.369988299 & 0.317016112 & 1 & 1 \\
\hline 5 & 0.75309088 & 0.866822163 & 0.523453221 & 0.565023619 & 2 & 2 \\
\hline 6 & 0.603807663 & 0.620439622 & 0.585688802 & 0.585218663 & 2 & 2 \\
\hline 7 & 0.833143565 & 0.629507422 & 0.278184995 & 0.343708098 & 1 & 1 \\
\hline 8 & 0.692742485 & 0.978491415 & 0.654192881 & 0.398168922 & 2 & 2 \\
\hline 9 & 0.742052675 & 0.592646858 & 0.289624287 & 0.326558445 & 1 & 1 \\
\hline 10 & 0.62781314 & 0.365138462 & 0.485198477 & 0.283729817 & 1 & 2 \\
\hline$\vdots$ & $\vdots$ & $\vdots$ & $\vdots$ & $\vdots$ & $\vdots$ & $\vdots$ \\
\hline 70 & 0.367412382 & 0.590387248 & 0.692746959 & 0.62396661 & 3 & 3 \\
\hline 71 & 0.479555791 & 0.630052699 & 0.795747193 & 0.654957234 & 3 & 3 \\
\hline 72 & 0.318797905 & 0.290232035 & 0.376480055 & 0.324328504 & 3 & 3 \\
\hline 73 & 0.418720261 & 0.451631428 & 0.77908381 & 0.227788025 & 3 & 3 \\
\hline 74 & 0.29301287 & 0.321279436 & 0.545408461 & 0.366476514 & 3 & 3 \\
\hline 75 & 0.385226266 & 0.413714892 & 0.658865834 & 0.669631158 & 4 & 4 \\
\hline 76 & 0.545467491 & 0.539314756 & 0.760187187 & 0.786090732 & 4 & 3 \\
\hline 77 & 0.383687857 & 0.392311966 & 0.742113723 & 0.49908387 & 3 & 3 \\
\hline
\end{tabular}

Hospital, Southern Medical University. And the set of disease diagnoses is $D=\{$ Not successful pregnancy and no symptoms of OHSS, Successful pregnancy and no symptoms of OHSS, Not successful pregnancy and symptoms of OHSS, Successful pregnancy and symptoms of OHSS\}, and the set of symptoms is a universe of discourse $S=\{$ Basic Hormone FSH, Basic Hormone P, Basic Hormone E2, Basic Hormone T, BMI, Day of HCG E2, Day of HCG endometrial thickness\}, where each symptom is described as follows: membership function $\mu_{A}(x)$, non-membership function $v_{A}(x)$, and hesitancy function $\pi_{A}(x)$.

Applying the conventional distance measures on IFS and DIVIFS to medical diagnosis, we have the results in Table 6. From Table 6, we conclude that the most accurate results of medical diagnosis are from Xu's equation (15) when $k=0.5$, which is also only a special case of (35) when $C_{1}=C_{2}=C_{3}=$ $C_{4}=C_{6}=C_{8}=0$ and $C_{5}=C_{7}=1$. And in 77 individuals, $74 \%$ of all the women can be classified correctly. However, using (38), the recognition rate will be $83 \%$. Some results of medical diagnosis have been shown in Table 7.

\section{Conclusion}

We propose a method for the evaluation of a degree of agreement in a group of individuals by calculating distances between DIVIFS. By analyzing the variation on the degree of hesitancy, we introduce a novel DIVIFS method derived from IVIFS, which is an effective method for us to construct dynamic IVIFS model from IFS and IVIFS. And then we propose a novel ranking condition for the distance measures on IFS, IVIFS, and DIVIFS. According to the new condition, we show the construction of distance measures on DIVIFS in theory. Finally, we apply some distances on DIVIFS to medical diagnosis based on pattern recognition. The experimental results show that the DIVIFS method and its distance measures are more comprehensive and more effective than the conventional distance measures on IFS and IVIFS.

\section{Acknowledgments}

This paper is funded by the National Natural Science Foundation of China (Grant no. 71271061, Grant no. 70801020), 
the "Twelfth Five-Years" Philosophy and Social Sciences Planning Project of Guangdong Province (Grant no. GD12XGL14), the "Twelfth Five-Year" Education Planning Project of Guangdong Province (Grant no. 2012JK129), Major Education Foundation of Guangdong University of Foreign Studies (Grant no. GYJYZDA12011), Business Intelligence Key Team of Guangdong University of Foreign Studies (Grant no. TD1202), Science and Technology Planning Project of Guangdong Province (Grant no. 2010B010600034), and High-Level Scientific Research Project of the Department of Education of Guangdong Province (Grant no. 396GK100017).

\section{References}

[1] L. A. Zadeh, "Fuzzy sets," Information and Computation, vol. 8, pp. 338-353, 1965.

[2] X. Li, R. Zhao, and W. Tang, "Similarity measures between fuzzy variables and their application to pattern ecognition," Information, vol. 13, no. 1, pp. 47-55, 2010.

[3] W. Tang, C. Wang, and R. Zhao, "Fuzzy parametric statistical inference," Information, vol. 14, no. 1, pp. 17-27, 2011.

[4] X. Li, L. X. Yang, and J. W. Gao, "Fuzzy edmundson-madansky inequality and its application to portfolio selection problems," Information, vol. 13, pp. 1163-1173, 2010.

[5] R. R. Yager, "Fuzzy modeling for intelligent decision making under uncertainty," IEEE Transactions on Systems, Man, and Cybernetics B, vol. 30, no. 1, pp. 60-70, 2000.

[6] K. T. Atanassov, "Intuitionistic fuzzy sets," Fuzzy Sets and Systems, vol. 20, no. 1, pp. 87-96, 1986.

[7] R. R. Yager, "Some aspects of intuitionistic fuzzy sets," Fuzzy Optimization and Decision Making, vol. 8, no. 1, pp. 67-90, 2009.

[8] E. Szmidt and J. Kacprzyk, "A similarity measure for intuitionistic fuzzy sets and its application in supporting medical diagnostic reasoning," in Proceedings of the 7th International Conference on Artificial Intelligence and Soft Computing (ICAISC '04), vol. 3070 of Lecture Notes in Artificial Intelligence, pp. 388393, June 2004.

[9] E. Szmidt and J. Kacprzyk, "Distances between intuitionistic fuzzy sets and their applications in reasoning," Studies in Computational Intelligence, vol. 2, pp. 101-116, 2005.

[10] E. Szmidt and J. Kacprzyk, "Dilemmas with distances between intuitionistic fuzzy sets: straightforward approaches may not work," Studies in Computational Intelligence, vol. 109, pp. 415430, 2008.

[11] V. Khatibi and G. A. Montazer, "Intuitionistic fuzzy set vs. fuzzy set application in medical pattern recognition," Artificial Intelligence in Medicine, vol. 47, no. 1, pp. 43-52, 2009.

[12] T. Chaira, "Intuitionistic fuzzy segmentation of medical images," IEEE Transactions on Biomedical Engineering, vol. 57, no. 6, pp. 1430-1436, 2010.

[13] D. K. Iakovidis and E. Papageorgiou, "Intuitionistic fuzzy cognitive maps for medical decision making," IEEE Transactions on Information Technology in Biomedicine, vol. 15, no. 1, pp. 100107, 2011.

[14] L. Dengfeng and C. Chuntian, "New similarity measures of intuitionistic fuzzy sets and application to pattern recognitions," Pattern Recognition Letters, vol. 23, no. 1-3, pp. 221-225, 2002.

[15] Y. Li, D. L. Olson, and Z. Qin, "Similarity measures between intuitionistic fuzzy (vague) sets: a comparative analysis," Pattern Recognition Letters, vol. 28, no. 2, pp. 278-285, 2007.
[16] Z. Xu, "Some similarity measures of intuitionistic fuzzy sets and their applications to multiple attribute decision making," Fuzzy Optimization and Decision Making, vol. 6, no. 2, pp. 109-121, 2007.

[17] Z. S. Xu and J. Chen, "An overview of distance and similarity measures of intuitionistic fuzzy sets," International Journal of Uncertainty, Fuzziness and Knowledge-Based Systems, vol. 16, no. 4, pp. 529-555, 2008.

[18] Z. Xu and R. R. Yager, "Intuitionistic and interval-valued intuitionistic fuzzy preference relations and their measures of similarity for the evaluation of agreement within a group," Fuzzy Optimization and Decision Making, vol. 8, no. 2, pp. 123-139, 2009.

[19] Z. Xu, "On similarity measures of interval-valued intuitionistic fuzzy sets and their application to pattern recognitions," Journal of Southeast University, vol. 23, no. 1, pp. 139-143, 2007.

[20] M. Xia and Z. Xu, "Some new similarity measures for intuitionistic fuzzy values and their application in group decision making," Journal of Systems Science and Systems Engineering, vol. 19, no. 4, pp. 430-452, 2010.

[21] G. Wei, "Some induced geometric aggregation operators with intuitionistic fuzzy information and their application to group decision making," Applied Soft Computing Journal, vol. 10, no. 2, pp. 423-431, 2010.

[22] K. Atanassov and G. Gargov, "Interval valued intuitionistic fuzzy sets," Fuzzy Sets and Systems, vol. 31, no. 3, pp. 343-349, 1989.

[23] X. H. Yuan, H. X. Li, and K. B. Sun, "Theory based on intervalvalued level cut sets of Zadeh fuzzy sets," Fuzzy Information and Engineering, vol. 2, pp. 501-510, 2009.

[24] Y. Zhang, P. Ma, and X. Su, "Pattern recognition using intervalvalued intuitionistic fuzzy set and its similarity degree," in Proceedings of the IEEE International Conference on Intelligent Computing and Intelligent Systems (ICIS '09), pp. 361-365, November 2009.

[25] D. F. Li, "Mathematical-programming approach to matrix games with payoffs represented by atanassovs interval-valued intuitionistic fuzzy sets," IEEE Transactions on Fuzzy Systems, vol. 18, no. 6, pp. 1112-1128, 2010.

[26] Z. H. Zhang, J. Y. Yang, Y. P. Ye, Y. Hu, and Q. S. Zhang, "Intuitionistic fuzzy sets with double parameters and its application to dynamic multiple attribute decision making," Information, vol. 15, no. 6, pp. 2479-2486, 2012.

[27] Z. Zhang, J. Yang, Y. Ye, Y. Hu, and Q. Zhang, "A scoring function of intuitionistic fuzzy sets with double parameters and its application to multiple attribute decision making," Information, vol. 15, no. 11A, pp. 4443-4450, 2012.

[28] Z. H. Zhang, J. Y. Yang, Y. P. Ye, Y. Hu, and Q. S. Zhang, "Intuitionistic fuzzy sets with double parameters and its application to pattern recognition," Information Technology Journal, vol. 11, no. 3, pp. 313-318, 2012.

[29] Q. Zhang, H. Yao, and Z. Zhang, "An interval-valued fuzzy reasoning approach based on weighted similarity measure," Advanced Materials Research, vol. 143-144, pp. 161-165, 2011.

[30] E. Szmidt and J. Kacprzyk, "Distances between intuitionistic fuzzy sets," Fuzzy Sets and Systems, vol. 114, no. 3, pp. 505-518, 2000.

[31] P. Grzegorzewski, "Distances between intuitionistic fuzzy sets and/or interval-valued fuzzy sets based on the Hausdorff metric," Fuzzy Sets and Systems, vol. 148, no. 2, pp. 319-328, 2004. 
[32] W. L. Hung and M. S. Yang, "Similarity measures of intuitionistic fuzzy sets based on Hausdorff distance," Pattern Recognition Letters, vol. 25, no. 14, pp. 1603-1611, 2004.

[33] W. Wang and X. Xin, "Distance measure between intuitionistic fuzzy sets," Pattern Recognition Letters, vol. 26, no. 13, pp. 20632069, 2005.

[34] H.-W. Liu, "New similarity measures between intuitionistic fuzzy sets and between elements," Mathematical and Computer Modelling, vol. 42, no. 1-2, pp. 61-70, 2005.

[35] C.-P. Wei, P. Wang, and Y.-Z. Zhang, "Entropy, similarity measure of interval-valued intuitionistic fuzzy sets and their applications," Information Sciences, vol. 181, no. 19, pp. 42734286, 2011. 


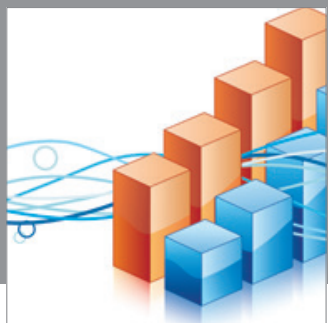

Advances in

Operations Research

mansans

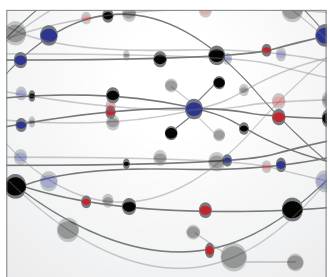

The Scientific World Journal
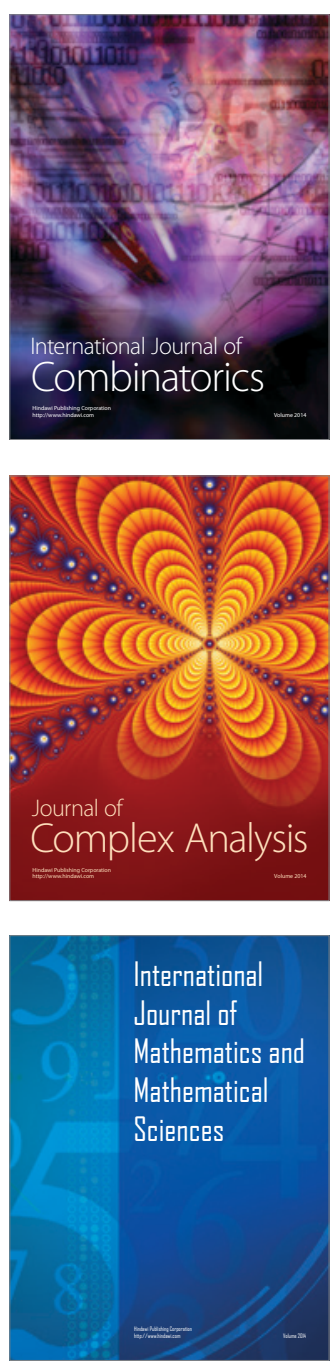
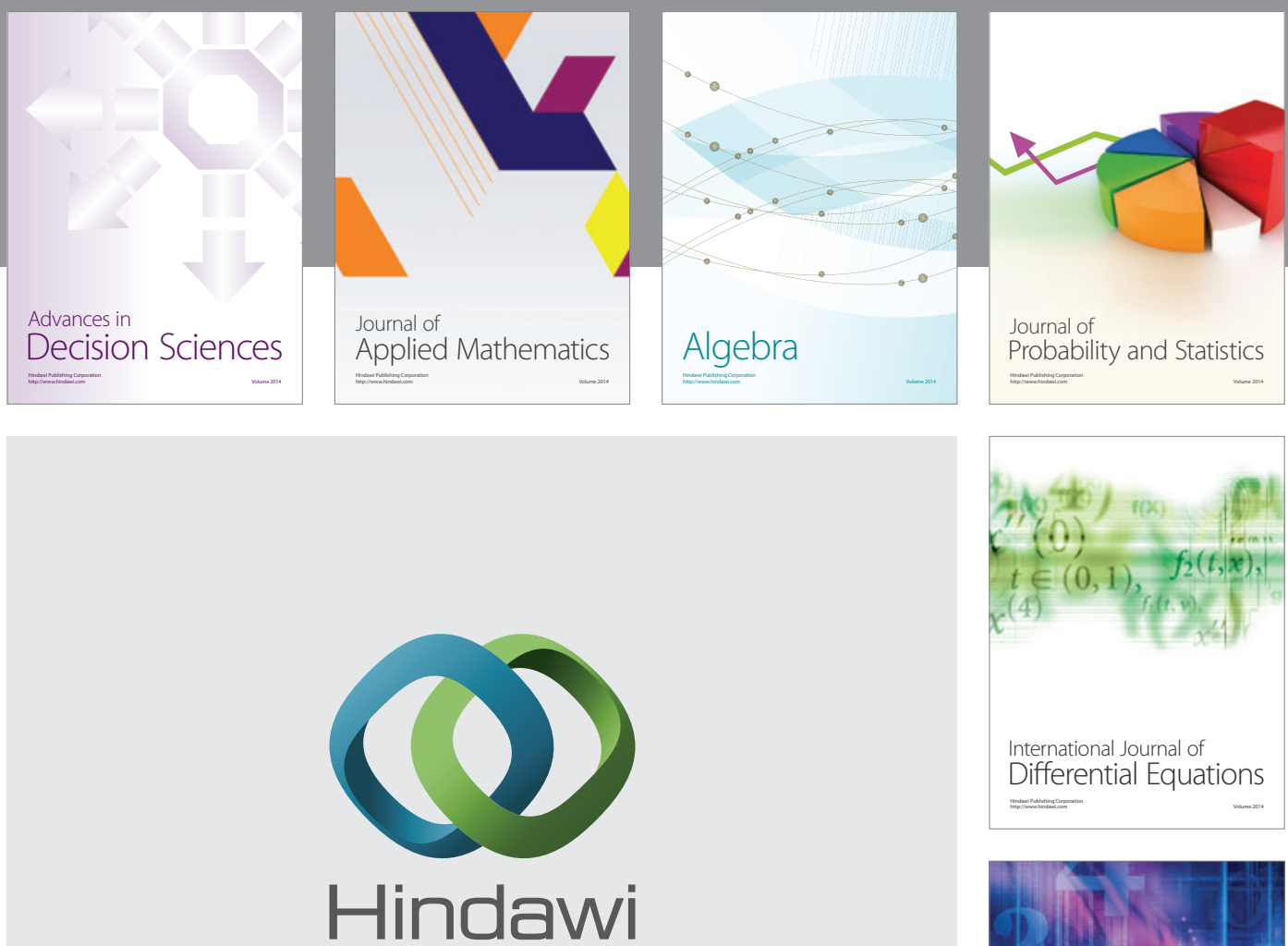

Submit your manuscripts at http://www.hindawi.com
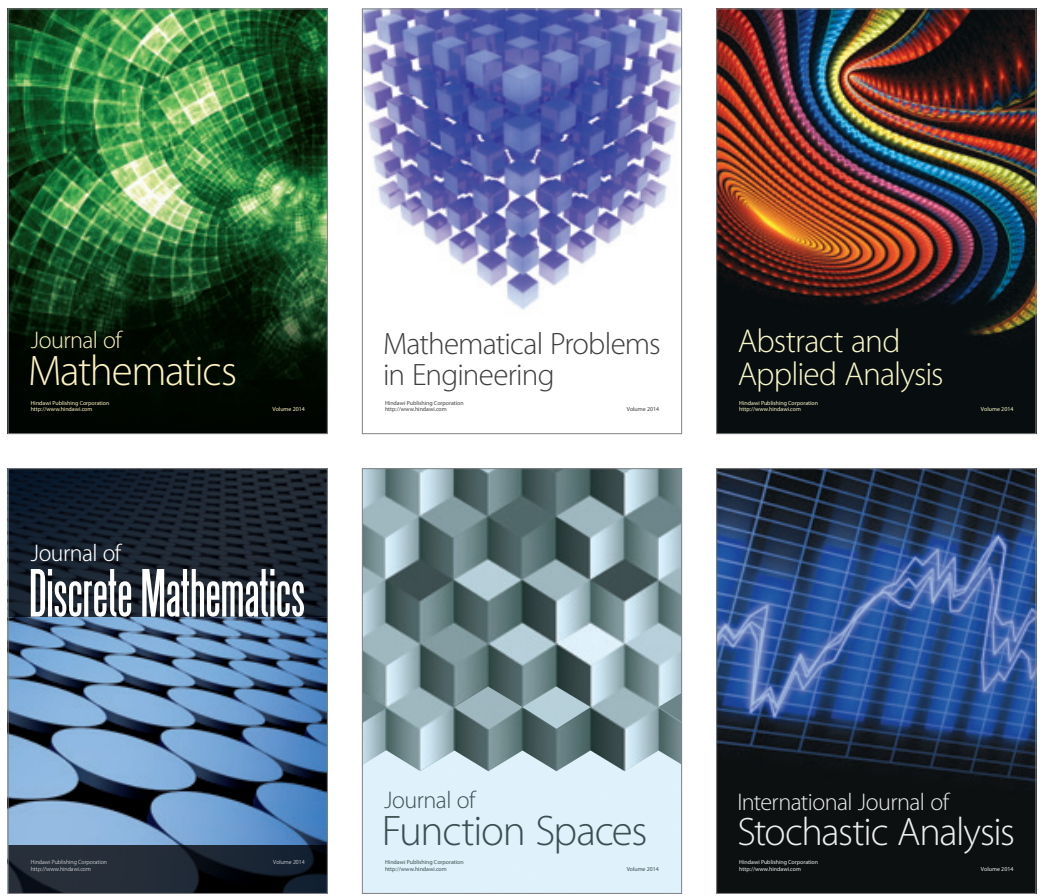

Journal of

Function Spaces

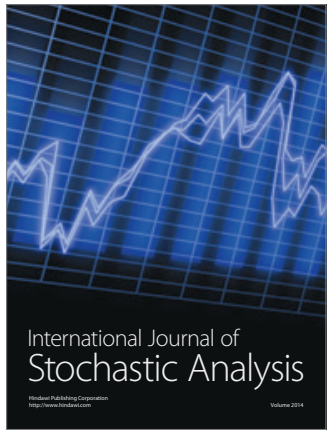

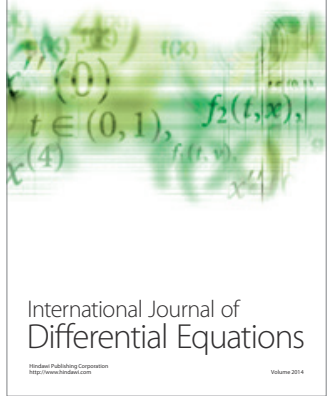
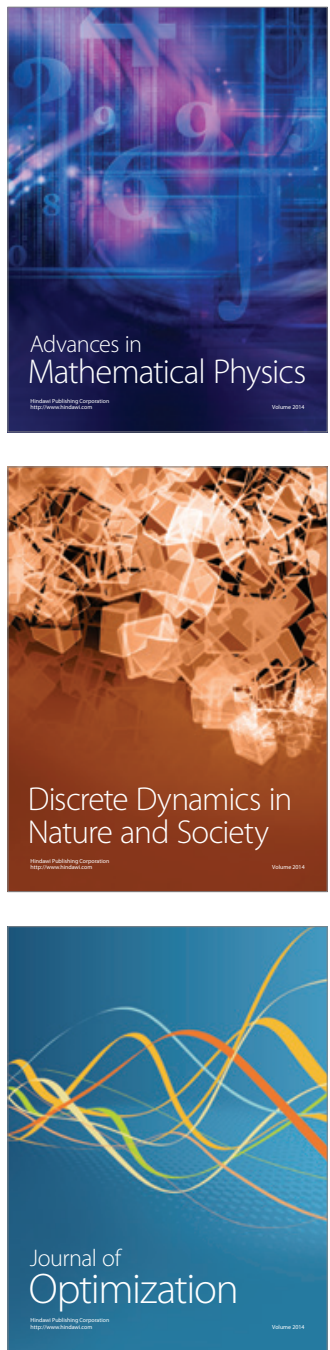